

\section{Longitudinal tracing of neurochemical metabolic disorders in working memory neural circuit and optogenetics modulation in rats with vascular cognitive impairment}

Huawei Lin

Fujian University of Traditional Chinese Medicine

\section{Tingting Jin}

Fujian University of Traditional Chinese Medicine

\section{Lewen Chen}

Fujian University of Traditional Chinese Medicine

\section{Yaling Dai}

Fujian University of Traditional Chinese Medicine

\section{Weiwei Jia}

Fujian University of Traditional Chinese Medicine

\section{Xiaojun He}

Fujian University of Traditional Chinese Medicine

\section{Minguang Yang}

Fujian University of Traditional Chinese Medicine

Jianhong Li

Fujian University of Traditional Chinese Medicine

\section{Shengxiang Liang}

Fujian University of Traditional Chinese Medicine

Jinsong Wu

Fujian University of Traditional Chinese Medicine

Jia Huang

Fujian University of Traditional Chinese Medicine

\section{Lidian Chen}

Fujian University of Traditional Chinese Medicine

Weilin Liu

Fujian University of Traditional Chinese Medicine Jing Tao ( $\sim$ taojing01@fjtcm.edu.cn )

Fujian University of Traditional Chinese Medicine 


\section{Research article}

Keywords: Chronic cerebral ischemia, Vascular cognitive impairment, Working memory, Neural circuit, Magnetic resonance spectroscopy, Neurochemical metabolism

Posted Date: November 3rd, 2020

DOl: https://doi.org/10.21203/rs.3.rs-100201/v1

License: (c) (i) This work is licensed under a Creative Commons Attribution 4.0 International License.

Read Full License

Version of Record: A version of this preprint was published at Brain Research Bulletin on May 1st, 2021. See the published version at https://doi.org/10.1016/j.brainresbull.2021.02.013. 


\section{Abstract}

Objective: Chronic cerebral ischemia leads to vascular cognitive impairment ( $\mathrm{VCl}$ ) that exacerbates along with ischemia time and eventually develops into dementia. Recent advances in molecular neuroimaging contribute to understand its pathological characteristics. We previously traced the anisotropic diffusion of water molecules suggests that chronic cerebral ischemia leads to irreversible progressive damage to white matter integrity. However, the abnormalities of gray matter activity following chronic cerebral ischemia remains not entirely understood.

Methods: In this study, in vivo hydrogen proton magnetic resonance spectroscopy ( ${ }^{1} \mathrm{H}-\mathrm{MRS}$ ) was applied to longitudinally track the neurochemical metabolic disorder of gray matter associated with working memory, and optogenetics modulation of neurochemical metabolism was performed for targeted treatment of $\mathrm{VCl}$.

Results: The results showed that the concentration of N-acetylaspartate (NAA) in the right hippocampus, left hippocampus, right medial prefrontal cortex (MPFC) and mediodorsal thalamus was decreased as early as 7 days after chronic cerebral ischemia, subsequently gamma-aminobutyric acid (GABA) declined whereas myo-inositol ( $\mathrm{ml}$ ) and glutamate (Glu) increased at 14 days, as well as choline (Cho) lost at 28 days, concurrently the change of Glu and GABA in the MPFC and hippocampus was ischemia timedependent manner within 1 month. Behaviorally, working memory and object recognition memory were impaired at 14 days, 28 days that significantly correlated with neurochemical metabolic disorders. Interestingly, using optogenetics modulation of PV neurons in the MPFC, the metabolic abnormalities of NAA and GABA in working memory neural circuit could be repaired after chronic cerebral ischemia, together with behavior improvements.

Conclusions: These findings suggested that as early as $1 \sim 4$ weeks after chronic cerebral ischemia, the metabolism of NAA, Glu, $\mathrm{ml}$ and Cho was synchronously impaired in neural circuit of hippocampusmediodorsal thalamus-mPFC, and the loss of GABA delayed in the hippocampus, and optogenetics modulation of parvalbumin (PV) neurons in the mPFC can improve the neurochemical metabolism of working memory neural circuit and enhance working memory.

\section{Background}

Chronic cerebral ischemia is a very common brain disease in middle-aged and elderly people, usually triggered by vascular disorders, thrombosis, or cardiac functions weaken(ladecola et al., 2019), which occurs hypoperfusion of brain blood flow unable to meet metabolic demand, limits oxygen and other nutrients supply and leads to the necrosis of brain tissue, cerebral atrophy or cerebral infarction, often involving cortical and subcortical important brain areas(Jellinger, 2008; Yang et al., 2017). Behaviorally, chronic cerebral ischemia causes a progressive vascular cognitive impairment ( $\mathrm{VCl}$ ) syndrome from mild cognitive impairment to dementia, which is mainly manifested as dysfunctions of attention, working memory, executive function and learning memory(O'Brien and Thomas, 2015). Vascular dementia (VD) is 
claimed to be the second most common cause of dementia after Alzheimer' disease, accounting for 15$30 \%$ of cases closely related to age(Goodman et al., 2017; van der Flier et al., 2018). The age-adjusted incidence of VD is 14.6 per 1,000 people and doubles every 5.3 years(Dichgans and Leys, 2017; Gorelick et al., 2011).

In the early stages of chronic cerebral ischemia, patients may not show obvious cognitive and daily activities disorders, missing the pathological diagnosis and prevention, however, in animal models, the substantial changes such as abnormal neuronal activity and functional connection and white matter lesions have been clearly observed as early as $2 \sim 10$ days after cerebral ischemic hypoperfusion(Liang et al., 2020; Soria et al., 2013; Tomimoto et al., 2003; Wang et al., 2015). Growing evidence suggests that metabolic abnormalities of neurochemicals is also one of the important risk factors for $\mathrm{VCl}$, and these metabolic disorders may occur earlier than structural abnormality and cognitive dysfunctions(Jones and Waldman, 2004; Venkat et al., 2015). Neurochemical refers to small organic molecule or peptide, as a neurotransmitter or neuromodulator or neuropeptide that participates in neural activity. Studies showed that chronic cerebral ischemia results in the decrease of neurochemicals and the imbalance of neurochemicals metabolism in the brain, which is closely related to the impairments of working memory and executive function(Galisova et al., 2014; Gasparovic et al., 2013). The molecular neuroimaging has been widely used to identify brain pathology changes associated with cognitive disorders. As a noninvasive molecular neuroimaging examination, hydrogen proton magnetic resonance spectroscopy $\left({ }^{1} \mathrm{H}\right.$ MRS) can detect the concentration of neurochemicals and metabolites in specific brain tissues. Evidence from clinical and animal studies has shown that cerebral ischemia leads to the loss of neurochemicals and metabolic abnormalities, including N-acetylaspartate (NAA), choline (Cho), lactic acid (Lac), glutamine (Gln), glutamate (Glu), gamma-aminobutyric acid (GABA) and myo-inositol (ml), etc., indicating abnormal activity of neurons and glial cells, dysfunction of neurotransmitters release and imbalance of excitation/inhibition accompanied by cerebral ischemia(Galisova et al., 2014; He et al., 2018; Hu et al., 2018; Yang et al., 2012). Therefore, neurochemicals and metabolic abnormalities are considered to be one of the pathogenesis in chronic cerebral ischemia-induced VCI and VD, and may be developed as potential clinical diagnostic indicators and therapeutic targets, but they remain poorly understood in terms of the pattern of changes in the occurrence and progression of disease, especially in the neural circuits associated with cognitive function.

Working memory impairment is one of the main symptoms of VCl and VD dysfunction(McGuinness et al., 2010). Working memory is a limited-capacity memory system and operating platform that temporarily stores and processes information in the process of performing cognitive tasks(D'Esposito and Postle, 2015). Initially, the prefrontal lobe was identified as the core brain area of working memory(GoldmanRakic, 1995). Subsequently, the interactions between the medial prefrontal cortex (mPFC) and hippocampus were revealed to involve in working memory, anatomically, the hippocampus has a robust projecting neural circuit to the MPFC, including the infralimbic and prelimbic cortices in rodents(Finn et al., 2019; Varela et al., 2014). More recently, increasing evidence has emerged that an indirect neural circuit from the hippocampus to mPFC via mediodorsal thalamic nucleus(Bolkan et al., 2017). During the 
working memory tasks, neuronal firing frequency and intensity has a significant increase in the mPFC and hippocampus, and the firing activity in MPFC is synchronized with theta oscillation of hippocampus(O'Neill et al., 2013; Tamura et al., 2017). Studies have demonstrated that the reduction of mediodorsal thalamus activity would lead to selective impairments in mPFC-dependent cognitive tasks, whereas enhancement of the mediodorsal thalamus to mPFC $\beta$-range frequency synchrony can improve the performance of working memory(Parnaudeau et al., 2013). Simultaneously, the release and circulation of neurotransmitters and neuromodulators, as well as the balance of their excitation and inhibition, are essential for these cognitive task dependent neural circuit activities. However, they are very sensitive to cerebral hypoxia and ischemia. In the early stages of ischemia, the concentration of some neurotransmitters or neuromodulators may be increased due to excessive excitation or decreased due to cell apoptosis or death. However, in the chronic phase, these neurochemicals are depleted or accumulation of metabolites in regulating the activities of neural circuit(Hu et al., 2018; Mattfeld and Stark, 2015). In this study, firstly a longitudinal ${ }^{1} \mathrm{H}-\mathrm{MRS}$ was used to investigate the dynamic changes of neurochemicals and metabolism in working memory neural circuit (including mPFC, hippocampus and mediodorsal thalamus) in a rat model of chronic cerebral ischemia, then optogenetics modulation of neurochemical metabolism was performed for targeted therapy, which provides theoretical and experimental basis for the clinical diagnosis and treatment of $\mathrm{VCl}$.

\section{Materials And Methods}

\subsection{Animal ethics and experimental design}

Thirty-one male Sprague-Dawley rats (8-week-old, weighing 250-300 g) were purchased from Shanghai SLAC Laboratory Animal Co., Ltd. (Shanghai, China). Experimental animals are raised by the Experimental Animal Center of Fujian University of Traditional Chinese Medicine [license number: SYXK (Min) 2019-0007]. Rats were reared on a 12-hour light/12-hour dark cycle and provided with food and water. All experimental procedures are strictly in accordance with the International Ethical Guidelines and the National Institutes of Health Guide for the Care and Use of Laboratory Animals, and were approved by the Ethics Committee of Fujian University of Traditional Chinese Medicine.

The experimental design was divided into two parts. In experiment 1, rats were randomly divided into the sham group $(n=17)$ and the permanent ligation of bilateral common carotid arteries $(2-\mathrm{VO})$ group $(n=$ 14), and observed for 28 days. Longitudinal ${ }^{1} \mathrm{H}$-MRS and working memory behavior were examined at 7 days, 14 days and 28 days following chronic cerebral ischemia.

In experiment 2, rats were randomly divided into the optogenetics stimulation group (OptoStim, $\mathrm{n}=10$ ) and the sham-optogenetics stimulation group (Sham-OptoStim, $n=10$ ), and treated for 21 days, ${ }^{1} \mathrm{H}-\mathrm{MRS}$, working memory behavior, and histology were examined after intervention.

\subsection{Establishment of a rat model of chronic cerebral ischemia}


The 2-VO method was performed to establish a rat model of chronic cerebral ischemia as previously described(Jin et al., 2017). All rats were fasted for 24 hours before surgery. They were anesthetized with $2.5 \%$ isoflurane gas during the operation, and vital signs such as breathing and heartbeat were monitored. First, we placed the anesthetized rat on a special surgical insulation pad, disinfected with $75 \%$ alcohol, and made a midline incision $(2.5 \times 2.5 \mathrm{~cm})$ along the skin to the neck. Then, the subcutaneous tissue was fully peeled to expose the trachea and we separated the common carotid arteries with micro forceps on both sides of the trachea. Next, pass an unabsorbable surgical suture between the common carotid arteries and the vagus nerve. Ligation the common carotid arteries for 5 minutes, after that, the other common carotid artery was ligated. Finally, we performed sutures, cleaned the wounds, and intraperitoneally injected 200,000 units/ $\mathrm{ml}$ of an animal penicillin sodium solution $(0.05 \mathrm{ml} / 100 \mathrm{~g})$. After the rats woke up, they were returned to the squirrel cage and given a normal diet. In the sham group, bilateral common carotid artery was just isolated and no ligation was performed.

\subsection{Optogenetics stimulation of parvalbumin neurons in the MPFC for modulating neurochemical metabolism after chronic cerebral ischemia}

\subsubsection{Stereotactic surgery}

Optogenetics stimulation of parvalbumin (PV) neurons in the mPFC was selected for treating $\mathrm{VCl}$. The OptoStim group and the sham-OptoStim group were injected optogenetics tracer viruses rAAV-fPV-cre-pAs and rAAV-Ef1a-DIO-hChR2-EYFP-WPRE-pA with $500 \mathrm{nl}$ per piece before the establishment of cerebral ischemia model, were packaged and purchased from (BrainTVA Co., Ltd, Wuhan, China) with 1:2 mixing ratio. First, the rats were anesthetized with $2.5 \%$ isoflurane and fixed to a brain stereotaxic instrument (RWD life science Co., Ltd, Shenzhen, China). Then, cut down their head hair, after exposing the front chimney, and the front vent was the coordinate origin, drilled a small hole above the inner frontal lobe (AP: $4.68 \mathrm{~mm}, \mathrm{ML}: 0.5 \mathrm{~mm}$, DV: $4 \mathrm{~mm}$ ), used a microsyringe pump at $40 \mathrm{nl} / \mathrm{min} .500 \mathrm{nl}$ virus was injected into the right medial prefrontal lobe of each group of rats. After the virus injection, the needle was kept in the brain for 5 minutes and then slowly withdrew. The optical fiber (Inper Co., Ltd, Hangzhou, China) ferrule was implanted into the corresponding brain area, and the wound was fixed and closed with dental cement.

\subsubsection{Optogenetics stimulation}

An optogenetics system (Plexon, Inc., Hong Kong) was used for activation of channelrhodopsin-2 (ChR2) and halorhodopsin (NpHR). The 473-nm blue light was delivered at $1 \mathrm{~mW}, 5 \mathrm{~ms}, 40 \mathrm{~Hz}$ in a square wave stimulation mode in the OptoStim group, and the 550-nm yellow light was delivered at $1 \mathrm{~mW}, 5 \mathrm{~ms}, 40 \mathrm{~Hz}$ in the sham-OptoStim group. The intervention started at 2 weeks after chronic cerebral ischemia, once a day, 15 minutes each time and lasting for 3 weeks.

\subsection{In vivo magnetic resonance angiography and in vivo ${ }^{1} \mathrm{H}-\mathrm{MRS}$}

A 7.0T small animal MRI scanner (Germany Bruker Biospec 70/20 USR) was used for the 3D time-of-fight (3D TOF) magnetic resonance angiography and ${ }^{1} \mathrm{H}$-MRS scan. First, the mice were anesthetized with 
$2.5 \%$ isoflurane, and then injected with $1 \%$ medetomidine. A custom-made holder including tooth bar and ear bar was used to minimize head motion, and then the surface coil was placed and fixed on the top of the rat's head, and then rats were sent to the receiving coil for scanning. During the scan, anesthesia was maintained with $1 \%$ isoflurane, and we used water circulation heating to maintain rat temperature and detect respiration at any time.

A 3D TOF magnetic resonance angiography scan was performed following parameters: Repetition Time $(T R)=15 \mathrm{~ms}$, Echo Time $(T E)=2.7 \mathrm{~ms}$, Field of view $(F O V)=35 \times 44 \times 24 \mathrm{~mm}$, Averages $=1$, Slices $=1$, Slice Thickness $=24 \mathrm{~mm}$, Time $=5 \mathrm{~min} 5 \mathrm{~s} 280 \mathrm{~ms}$. T2WI scans were acquired using rapid acquisition with relaxation enhancement pulse sequence with the following parameters: $T R=4200 \mathrm{~ms}, \mathrm{TE}=35 \mathrm{~ms}$, FOV $=32 \times 32 \mathrm{~mm}$, Averages = 2, Slices $=21$, Slice Thickness $=1 \mathrm{~mm}$, Time $=4 \mathrm{~min} 28 \mathrm{~s} 800 \mathrm{~ms}$.

For ${ }^{1} \mathrm{H}-\mathrm{MRS}$, the right hippocampus, left hippocampus, right mPFC and mediodorsal thalamus were selected as regions of interest (ROI) and delineated in a T2-weighted image with a scan volume of $20 \times$ $20 \times 20$ mm (Supplementary Fig. S1-S4). Point resolved selective sequence was used for signal acquisition, with TR $=1500 \mathrm{~ms}$, TE $=20 \mathrm{~ms}$ and Time $=6 \mathrm{~min} 24 \mathrm{~s}$.

After the data collection was completed, spectral image analysis and data processing were performed using Topspin 3.0 software(Xi et al., 2011). The metabolite area under the peak was used to quantify different neurochemical metabolites. In order to reduce the individual differences between animals, we use the creatine $(\mathrm{Cr})$ peak as the internal spectral reference. The ratios of $\mathrm{NAA} / \mathrm{Cr}, \mathrm{ml} / \mathrm{Cr}, \mathrm{Cho} / \mathrm{Cr}$, Glu/Cr and GABA/Cr at different time points in each brain region were used for statistical analysis. Each MRS metabolite was identified by its part per million ( $\mathrm{ppm}$ ) position of the nuclear spectrum, including, $\mathrm{ml}$ 3.56, Cho 3.20 ppm, Cr 3.05 ppm, NAA 2.02-2.05 ppm, Glu 2.1-2.5 ppm and Glu 2.28 ppm(Zhou et al., 2013).

\section{5 $\mathrm{Y}$ maze test}

The $Y$ maze consists of three $(A, B, C)$ arms of equal length $(50 \mathrm{~cm} \times 10 \mathrm{~cm} \times 20 \mathrm{~cm})$ and were placed at 120-degree angles. We use the spontaneous alternating experimental protocol to test the working memory of rats. Rats was placed at the edge of one maze and allowed to explore freely for 8 min after a habituation phase of $30 \mathrm{~s}$. An arm entry was scored when a rat placed four paws within that arm. And when the rat entered the different arms three times in succession (e.g., $A-B-C, C-A-B$ or $B-C-A$ ), we count as completing a complete spontaneous alternation. Our outcome index is alternating rate $=$ correct number of alternations/(total number of times to enter the arm-2) $\times 100$, representing the correct degree of alternation in the $Y$ maze(Sohn et al., 2019).

\subsection{Novel object recognition test (NOR)}

The device for NOR includes a black coverless cuboid $(80 \mathrm{~cm} \times 80 \mathrm{~cm} \times 60 \mathrm{~cm}$ ), and 3 solid objects, where "A" and "a" are exactly the same, and "B" is a novel object. The experimental process is divided into 3 stages: habituation, training and testing(Rutten et al., 2008). In the habituation phase, rats were allowed to explore freely in an open box for 10 minutes and then returned to the original cage. Before the next 
experiment, the box was wiped with $75 \%$ disinfectant alcohol to remove residual odor. In the training phase, two identical objects "A" and "a" were placed in the two corners of the black open box, rats were released in the middle of the opposite wall with its back to the objects, and allowed to explore the area for 10 minutes. The test phase occurred after $1 \mathrm{~h}$ and $24 \mathrm{~h}$ : the memory retrieval test was performed on the rats at $1 \mathrm{~h}$ after learning, the object " $\mathrm{A}$ " remained unchanged, the position of the object "a" was replaced by the object "B", and rats were placed in the box with its back to the object and allowed to explore freely for $5 \mathrm{~min}$. At $24 \mathrm{~h}$ after the learning phase, rats were also put into the box as the same way and allowed to explore freely for $5 \mathrm{~min}$. The movement track of rats and the exploration time of "A" and "B" objects were recorded by camera, respectively as TA and TB. Our outcome index is recognition index $=T B /(T A+T B)$, which represents the degree of discrimination of novel objects.

\subsection{Immunofluorescence and Immunohistochemistry}

The rats were anesthetized by intraperitoneally injection of $3 \%$ pentobarbital sodium $(0.33 \mathrm{ml} / 100 \mathrm{~g}$ body weight), the chest was opened, the cardiac was poured into $0.9 \%$ physiological saline and perfusion fixed by $4 \%$ paraformaldehyde, then decapitated and brain was removed. For immunofluorescence observation of optogenetics virus expression, the brain tissues were fixed with $4 \%$ paraformaldehyde for $24 \mathrm{~h}$. $30 \mu \mathrm{m}$ thick coronal slices were cut using a vibrating microtome (Leica, VT1000S, Germany). Then washed the slices in PBS, incubated with $0.3 \%$ Triton X-100 for 30 minutes, blocked with $3 \%$ bovine serum albumin for 2 hours, and incubated with anti-Cre antibody (1:200; ab190177; Abcam, Cambridge) at $4^{\circ} \mathrm{C}$ overnight. The next day, the sections were washed in PBS and incubated with a secondary antibody Alexa Fluor 488 conjugated goat anti-rabbit IgG (1:500, A-11008, Invitrogen, USA) at $37^{\circ} \mathrm{C}$ for $2 \mathrm{~h}$. Then the immunofluorescence in the mPFC was observed under the confocal laser microscope (ZEISS, Inc., German). For immunohistochemical detection of glutamate decarboxylase 67 (GAD67) and N-methyl-Daspartic acid receptor (NMDAR) expression, the brain tissues were fixed with $4 \%$ paraformaldehyde for $24 \mathrm{~h}$, embedded in paraffin to prepare $5 \mu \mathrm{m}$ coronal sections. After the citric acid antigen was repaired, we stained according to the steps of the immunohistochemistry kit (MX Biotechnologies Co., Ltd, Fuzhou, China). Added primary anti-GAD67 (1:200; ab25116; Abcam, Cambridge) and anti-NMDAR (1:200; ab52177; Abcam, Cambridge) dropwise, and then put them at $4^{\circ} \mathrm{C}$ overnight. On the second day, the secondary antibody and DAB developer were added dropwise, and the slide was mounted after hematoxylin staining. Each rat selected 4 fields of vision using the Image $\mathrm{J}$ pathological image analysis system to calculate the average optical density values of GAD67 and NMDAR in the mPFC region.

\subsection{Statistical analysis}

All research data were statistically analyzed using SPSS 20.0 software (SPSS, Armonk, NY, USA) and as Sharpiro-Wilk tests showed normality distributions for neurochemical metabolism and behavior test at a single time point, data were presented as mean \pm standard deviation. Considering that ${ }^{1} \mathrm{H}-\mathrm{MRS}$ and behavioral test data were acquired at three time points, we used repeated measures analysis of variance (ANOVA). The Mauchly's test was used for the assumption of sphericity, and the Bonferroni method was performed for the pairwise comparisons following significant overall results. Pearson and Spearman correlation analysis methods were performed for correlation analysis between ${ }^{1} \mathrm{H}$-MRS and behavioral 
test. Independent samples t-test was performed for the comparison of behavior tests, neurochemicals and immunohistochemistry. $P<0.05$ was considered to indicate a statistically significant difference.

\section{Results}

\subsection{Magnetic resonance angiography of bilateral common carotid arteries in rats with chronic cerebral ischemia}

To identify whether the successful of the 2-VO model of chronic cerebral ischemia, 3D TOF MR angiography was used to detect the diameter of bilateral common carotid arteries at 24 hours after 2-VO operation. Compared with the sham group, the bilateral common carotid arteries of the 2-VO model rats were obviously blocked, and there were new small vessels, suggesting that the rat model of chronic cerebral ischemia was successfully established (Supplementary Fig. S5).

\subsection{Longitudinal tracking of neurochemicals and metabolism in the working memory neural circuit of rats with chronic cerebral ischemia}

To explore the time course changes of neurochemicals and metabolism in working memory neural circuit following chronic cerebral ischemia, the ${ }^{1} \mathrm{H}$-MRS was performed to examine the concentration of NAA, ml, Cho, Glu and GABA of hippocampus, mPFC and mediodorsal thalamus in rats with sham or 2-VO operation and the $\mathrm{Cr}$ peak is frequently used as an internal reference(Krahe et al., 2020). Figure 1A-1F showed that in the right hippocampus, the concentration of NAA was significantly decreased at 7 days, 14 days and 28 days following chronic cerebral ischemia $(P<0.01$ or $P<0.001)$, and $\mathrm{ml}$ and Glu were significantly increased at 14 days and 28 days $(P<0.05$ or $P<0.01$ or $P<0.001)$, Cho and GABA were significantly decreased at 28 days $(P<0.01$ or $P<0.001)$. Moreover, the concentration of NAA and GABA at 14 days and 28 days was significantly lower than that at 7 days following chronic cerebral ischemia $(P<0.05$ or $P<0.001)$, whereas Glu was higher $(P<0.01$ or $P<0.001)$.

In the left hippocampus, Fig. 2A-2F showed similar results with the right hippocampus, except the concentration of $\mathrm{ml}$ at 14 days and 28 days was also significantly lower than that at 7 days following chronic cerebral ischemia $(P<0.05$ or $P<0.01)$.

In the right mPFC, Fig. 3A-3F showed that the concentration of NAA and $\mathrm{ml}$ was significantly decreased and increased at 7 days, 14 days and 28 days following chronic cerebral ischemia, respectively $(P<0.05$ or $P<0.01$ or $P<0.001)$, and Glu and GABA were significantly increased and decreased at 14 days and 28 days, respectively $(P<0.05$ or $P<0.01$ or $P<0.001)$, and Cho was significantly decreased at 28 days $(P<$ 0.05). Moreover, the concentration of NAA and GABA at 14 days and 28 days were significantly lower than that at 7 days $(P<0.01$ or $P<0.001)$, and $\mathrm{ml}$ and Glu at 28 days were significantly higher than that at 7 days following chronic cerebral ischemia $(P<0.05$ or $P<0.01)$.

In the mediodorsal thalamus, Fig. 4A-4F showed that the concentration of NAA was significantly decreased at 7 days, 14 days and 28 days following chronic cerebral ischemia $(P<0.05$ or $P<0.001)$, and 
$\mathrm{ml}$ and Glu were significantly increased at 14 days and 28 days $(P<0.05$ or $P<0.001)$, and GABA were significantly decreased at 14 days and 28 days $(P<0.05$ or $P<0.001)$. Moreover, the concentration of NAA and $\mathrm{ml}$ at 28 days was significantly lower and higher than that at 7 days, respectively $(P<0.05$ or $P$ $<0.01$ ), and Glu and GABA were significantly higher and lower at 14 days and 28 days than those at 7 days following chronic cerebral ischemia, respectively $(P<0.05$ or $P<0.001)$.

Therefore, these results suggested that NAA in the right hippocampus, left hippocampus, right mPFC and mediodorsal thalamus were initially decreased at 7 days after chronic cerebral ischemia, and GABA began to decline whereas $\mathrm{ml}$ and Glu began to increase at 14 days, as well as Cho began to decline at 28 days. Importantly, the increase of Glu, $\mathrm{ml}$ and the decrease of NAA, GABA in the mPFC and hippocampus were ischemia time-dependent manner within 1 month.

\subsection{Working memory and object recognition memory were impaired in rats with chronic cerebral ischemia}

The $Y$ maze was used to examine the working memory. Figure $5 \mathrm{~A}$ showed that there was no change in the correct alternation rate of the three arms in $Y$ maze at 7 days following chronic cerebral ischemia $(P>$ $0.05)$. However, at 14 days and 28 days, the alternation rate was decreased significantly $(P<0.001)$, as well as the alternation rate at $14 \mathrm{~d}$ and $28 \mathrm{~d}$ was significantly lower than that at 7 days $(P<0.05$ or $P<$ $0.001)$.

The NOR test was used to examine the object recognition memory. Figure $5 \mathrm{~B}, 5 \mathrm{C}$ showed that the recognition index of novel objects was intact at 7 days following chronic cerebral ischemia, similar to that of the sham operated rats $(P>0.05)$. However, at 14 days and 28 days, the recognition index of 1 hour and 24 hours test was significantly decreased $(P<0.001)$, and the recognition index at 14 days and 28 days was significantly lower than that at 7 days $(P<0.01$ or $P<0.001)$.

These results suggested that the working memory and object recognition memory were impaired in the early stage of chronic cerebral ischemia (within 1 month) and that progressively aggravated following ischemia time.

Furtherly, Pearson and Spearman correlation analysis results showed that the concentration of NAA and GABA in the right hippocampus was positively correlated with the alternation rate of $\mathrm{Y}$ maze $(\mathrm{r}=0.45$ and $0.32, P<0.01$ and $P<0.05)$, whereas Glu and $\mathrm{ml}$ were negatively correlated $(\mathrm{r}=-0.51$ and $-0.32, P<0.001$ and $P<0.05$, Fig. 5D-5E and Supplementary Table S1). In the left hippocampus, NAA and Cho was positively correlated with the alternation rate $(r=0.62$ and $0.33, P<0.001$ and $P<0.05)$, whereas Glu and $\mathrm{ml}$ were negatively correlated ( $\mathrm{r}=-0.36$ and $-0.36, P<0.05$, Fig. 5F and Supplementary Table S1). In the right mPFC, NAA and GABA were positively correlated with the alternation rate $(r=0.59$ and $0.39, P<$ 0.001 and $P<0.05)$, whereas Glu was negatively correlated $(r=-0.42, P<0.05$, Fig. 5G-5I and Supplementary Table S1). In the mediodorsal thalamus, GABA and Glu were positively correlated and negatively correlated with the alternation rate, respectively $(r=0.37$ and $-0.33, P<0.05$, Supplementary Table S1). In addition, we found that the metabolism of these neurochemicals in the hippocampus, mPFC and mediodorsal thalamus was also related to object recognition memory (Supplementary Table S1). 
These results indicated that abnormal neurochemical metabolism results in dysfunctions of working memory and object recognition memory after chronic cerebral ischemia.

\subsection{The neurochemical metabolism was improved by optogenetics modulation of PV neurons in the $\mathrm{mPFC}$ of rats with chronic cerebral ischemia}

To verify whether the regulation of neurochemical metabolism can repair the pathological changes after cerebral ischemia, optogenetics stimulation of PV neurons in the right MPFC was performed. The results showed that optogenetics viruses were successfully expressed in the MPFC of rats with chronic cerebral ischemia (Fig. 6A). After 3 weeks of optogenetics intervention, the working memory was improved $(P<$ 0.01 , Fig. $6 B$ ), and the concentration of NAA and GABA was increased in the right mPFC, right hippocampus and mediodorsal thalamus $(P<0.05$ or $P<0.01$ or $P<0.001$, Fig. 6C-6G and Supplementary Fig. S6-11), and $\mathrm{ml}$ was decreased $(P<0.05$, Fig. $6 \mathrm{C})$, as well as Glu had a decreasing trend in the right mPFC ( $P=0.053$, Fig. 6C). Additionally, the expression of NMDAR was decreased, whereas GAD67 was increased in the mPFC of rats with chronic cerebral ischemia $(P<0.05$, Fig. $6 \mathrm{H}-6 \mathrm{I})$. These results demonstrated that the metabolic abnormality of NAA, GABA and Glu in working memory neural circuit was repaired through optogenetics modulation of PV neurons in the MPFC of rats with chronic cerebral ischemia.

\section{Discussion}

In this study, the longitudinal experimental design was performed to track the pathological process of rats with chronic cerebral ischemia. ${ }^{1} \mathrm{H}$-MRS was applied to detect the dynamic changes of neurochemicals and metabolism in working memory neural circuit following chronic cerebral ischemia. The results showed that the concentration of NAA in the right hippocampus, left hippocampus, right MPFC and mediodorsal thalamus were initially decreased at 7 days after chronic cerebral ischemia, and GABA began to decline whereas $\mathrm{ml}$ and Glu began to increase at 14 days, as well as Cho began to lose at 28 days, concurrently the increase of Glu and the decrease of GABA in the MPFC and hippocampus were ischemia time-dependent manner. Behaviorally, working memory and object recognition memory were impaired in the early stage of chronic cerebral ischemia (within 1 month) and that progressively aggravated following cerebral ischemia time. Simultaneously, correlation analysis indicated that abnormal neurochemical metabolism results in dysfunctions of working memory and object recognition memory after chronic cerebral ischemia. Interestingly, the metabolic abnormality of NAA and GABA in working memory neural circuit was repaired and Glu has an improvement trend through optogenetics modulation of PV neurons in the MPFC of rats with chronic cerebral ischemia.

\subsection{The neural circuit of hippocampus-mediodorsal thalamus-mPFC orchestrates working memory}

The main clinical manifestations of $\mathrm{VCl}$ patients are poor performance in cognitive tasks controlled by frontal and subcortical, especially working memory and executive function, caused by reduced blood flow to the brain(Kertesz and Clydesdale, 1994; McGuinness et al., 2010; Venkat et al., 2015). Working memory 
is considered to be the central component of executive function, regulated by interactions between hippocampus and mPFC(Miller and Cohen, 2001; Preston and Eichenbaum, 2013). Lesion and electrophysiological studies suggest that the neural circuit of the ventral hippocampus projects directly to the mPFC is critical for successful task performance of working memory(Izaki et al., 2008; O'Neill et al., 2013). Moreover, during the memory maintenance phase, the direction of information flow is from the hippocampus to MPFC, while during the memory retrieval phase, the direction is from the MPFC to hippocampus(Dolleman-van et al., 2019; Eichenbaum, 2017). In recent years, studies have demonstrated that the mPFC exist mutual connectivity and communication with the hippocampus and mediodorsal thalamus and are essential for maintaining working memory(Bolkan et al., 2017; Kupferschmidt and Gordon, 2018; Parnaudeau et al., 2018; Schmitt et al., 2017). In the mediodorsal thalamus, there are glutamatergic thalamocortical neurons and GABAergic interneurons(Kuroda et al., 1998; Tiwari et al., 2013) that receive projection from mPFC neurons and then drive mPFC activity to integrate signals for adaptive goal-directed behavior(Miller et al., 2017). Therefore, the direct or indirect neural circuit of hippocampus-mediodorsal thalamus-mPFC orchestrates neural activity and communication that gives rise to working memory.

\subsection{The neurochemicals and metabolism were impaired in the early stage of chronic cerebral ischemia}

A growing number of studies have shown that chronic cerebral ischemia results in the lesions of the frontal lobe, hippocampus and thalamus, including neurochemicals and metabolic disorders(Chen et al., 2016; Galisova et al., 2014; He et al., 2018; Kalaria, 2016). In vivo ${ }^{1} \mathrm{H}-\mathrm{MRS}$ had been widely used to reveal the pathological characteristics and underlying mechanism of the neurochemicals and metabolic alterations in response to cerebral ischemia in humans and animals(Bruhn et al., 1989; Gurol, 2016; Igarashi et al., 2001). NAA is a specific amino acid, which is mainly synthesized in the mitochondria of neurons and exists in neuron cells and their axons(Tomaszewicz et al., 2003). It is widely considered as a marker of neuronal loss and injury, and is highly sensitive to cerebral ischemia(Bertolino et al., 2003; Demougeot et al., 2004; Jones and Waldman, 2004). Growing evidence also has shown that declined NAA is significantly associated with memory impairment(Jayaweera et al., 2015; Zhou et al., 2012). The present study showed that the concentration of NAA in the hippocampus, MPFC and mediodorsal thalamus were significantly decreased at 7 days, 14 days and 28 days after chronic cerebral ischemia, and working memory and object recognition memory were impaired at 14 days and 28 days, consistent with previous studies(Farkas et al., 2007; Han et al., 2019). Therefore, the neurochemical changes may precede cognitive impairments, suggesting that in vivo ${ }^{1} \mathrm{H}-\mathrm{MRS}$ has advantages in early pathological diagnosis of $\mathrm{VCl}$.

Glu is the main excitatory neurotransmitter in the brain and is essential for maintain the normal synaptic transmission and plasticity, but if the concentration of Glu exceeds the physiological range, then may cause excitatory neurotoxicity and damage neurons(Jun et al., 2014). While GABA is a major inhibitory neurotransmitter, which coordinates neural activity and produces a calming effect, but its concentration is too high or too low will cause damage to the nervous system(Marsman et al., 2017). It is widely observed that an imbalance between excitatory and inhibitory amino acids underlies cerebral ischemic 
damage(Bezalel et al., 2019; Marsman et al., 2017). In this study, it was found that there was no significant difference in the concentration of Glu and GABA within 1 week after chronic cerebral ischemia, but 2 weeks later, an obvious imbalance of excitation and inhibition was observed in detected hippocampus, mPFC and mediodorsal thalamus, of which Glu was gradually increased and GABA gradually decreased with ischemia time-dependent manner. Furthermore, the metabolic disorder of GABA in the MPFC and mediodorsal thalamus was occurred earlier than that in the hippocampus. Therefore, these suggest that the GABAergic neurons of the mPFC and mediodorsal thalamus and the glutamatergic neurons of the hippocampus were impaired in the working memory neural circuit in the early stage of chronic cerebral ischemia. Indeed, Glu and GABA are involved in working memory by regulating the functional connections of mPFC-hippocampus and mPFC-mediodorsal thalamus and synaptic plasticity(Auger and Floresco, 2014; Ferguson and Gao, 2018a; Huang et al., 2018; Thielen et al., 2018). Here, we found that the metabolism of Glu and GABA in the hippocampus, mPFC and mediodorsal thalamus was significantly correlated with the alternation rate of $Y$ maze and object recognition index, indicating that abnormal neurochemical metabolism results in dysfunctions of working memory and object recognition memory after chronic cerebral ischemia.

In addition, the results of this study showed that $\mathrm{ml}$ and Cho of the hippocampus, mPFC was increased and decreased at 14 days and 28 days after chronic cerebral ischemia, respectively. $\mathrm{ml}$ mainly exists in glial cells and is considered to be a marker of glial cells, which often appears to increase during the hyperplasia of glial cells and the activation of phospholipase(Glanville et al., 1989). Studies have found that reduced $\mathrm{ml}$ can be detected in animal models of cerebral ischemia(Hu et al., 2018; Jones and Waldman, 2004; Yang et al., 2012). The possible reason is that chronic ischemic stress in the brain tissues leads to reactive hyperplasia of glial cells, which response to inflammatory and result in increased $\mathrm{ml}$. Cho is an important acetylcholine neurotransmitter precursor compound. Glyceryl phosphate choline in the cytoplasm of the neuron and choline phosphate degraded from the phospholipid membrane of the neuron is the main components of the Cho wave(Klein, 2000). Under the action of acetylcholine transferase, Cho can be converted to acetylcholine and participate in neural information processing related to memory and recognition behaviors(Liu and Wang, 2016). It is reported that maintaining stable levels of acetylcholine in the frontal cortex and hippocampus are essential for the formation of memory(Stoiljkovic et al., 2015). Remarkably, there was still controversy on the changes of Cho in the brain of VCl patients. Studies suggested that VD patients present cholinergic deficits in the brain and cerebrospinal fluid that is closely related to the cognitive impairment(Wang et al., 2009). Other studies showed that the central cholinergic pathway may not participate in the pathological progression of $\mathrm{VCI}($ Bella et al., 2016). In this study, Cho in the mPFC and hippocampus was synchronously decreased at 28 days after chronic cerebral ischemia, but in the mediodorsal thalamus unchanged. Therefore, the loss of Cho in different brain areas was asynchronous and delayed in time after chronic cerebral ischemia.

\subsection{Optogenetics modulation of PV neuron in the mPFC can improve working memory in rats with VCl}

Optogenetics was an innovative type of neuromodulation technology with high spatial and temporal accuracy that used photosensitive ion channel ChR2 to activate neural activities with blue light, while 
neuronal inhibition results from illuminating NpHR with yellow light(Deisseroth, 2011; Deisseroth et al., 2006). Importantly, optogenetics has been applied to stimulate specific neuronal types can improve cognitive impairment(Park et al., 2020; Wang et al., 2019). As a typical GABAergic neuron, PV interneurons in the MPFC provided a inhibitory effect on excitatory pyramidal neurons, thereby optogenetics can significantly improve the balance of excitation and inhibition of mPFC by regulating the excitability of PV neurons(Ferguson and Gao, 2018b; Selimbeyoglu et al., 2017).

In addition, PV interneurons can participate in the generation of gamma oscillations, which is closely related to information processing and memory(Buzsáki and Draguhn, 2004). And a pair of studies have been conducted to silence or activate PV interneurons through optogenetics, and found that they are necessary and sufficient in generating and maintaining gamma oscillations(Cardin et al., 2009; Sohal et al., 2009).

Stimulation of PV interneurons drive rapidly discharge and short-term kinetics of synapses, generating gamma oscillations, and synchronously inhibit post-synaptic potential to local excitatory pyramidal neurons, which can enhance working memory(Hu et al., 2014). Studies have shown that vascular dementia disrupt gamma oscillation of MPFC that had been shown to be closely related to the working memory (Li et al., 2019; Xu et al., 2012).

Then, studies have shown that optogenetic stimulation of PV interneurons at $40 \mathrm{~Hz}$ can restore the gamma oscillation, and improve the object recognition ability and memory impairment in an AD mouse model.

This study was also applicable to $40 \mathrm{~Hz}$ optogenetics stimulation of PV interneurons in the right mPFC can enhance working memory in rats with $\mathrm{VCl}$. Therefore, optogenetics may be a potential treatment for working memory impairment of $\mathrm{VCl}$.

Similarly, this study found that optogenetics stimulation of PV interneurons in mPFC at $40 \mathrm{~Hz}$ could rescue the working memory in $\mathrm{VCl}$ rats. Therefore, optogenetics may be novel strategy for treating memory deficits in $\mathrm{VCl}$ models.

In addition, the study demonstrated that optogenetics modulation of PV neurons in the MPFC can improve the pathological changes in which the concentration of NAA and GABA in the right MPFC was significantly increased, $\mathrm{ml}$ was significantly reduced, and Glu had a tendency to decrease, simultaneously, the expression of NMDAR was decreased, whereas GAD67 was increased in the MPFC of rats with chronic cerebral ischemia. NMDAR was an important ionic Glu receptor that can mediate $\mathrm{Ca}^{2+}$ influx into the post-synaptic membrane and induce post-synaptic depolarization, which is the basis of synaptic plasticity(Rajani et al., 2020). Besides, GABA was synthesized by the decarboxylation of GAD, and then transported by the GABA vesicle transporter to synaptic vesicles, which activates the $G_{A B A}$ receptor to mediate $\mathrm{Cl}^{-}$flow to exert its inhibitory effect(Hwang et al., 2018). The expression of GAD67 were highly correlated with GABA levels(Lazarus et al., 2015), which are necessary for the maturation of inhibitory circuits in the cerebral cortex(Battaglioli et al., 2003; Esclapez et al., 1994). Therefore, optogenetics 
stimulation of PV neurons can promote the release of inhibitory neurotransmitters GABA, activing the expression of GAD67, and negative feedback regulation of excitatory neurons and reduced the expression of NMDAR. These suggested that the metabolic abnormality of GABA and Glu in working memory neural circuit was repaired through optogenetics modulation of PV neurons in the MPFC of rats with chronic cerebral ischemia.

\subsection{Limitations}

The change of neurochemical metabolism and behavior of rats with $\mathrm{VCl}$ was determined within 28 days following chronic cerebral ischemia, and further longer time periods should be observed. Considering the scanning time and anesthesia time of animals, the right mPFC was chosen to be detected by ${ }^{1} \mathrm{H}-\mathrm{MRS}$. We found that there was no difference in neurochemicals metabolism between the left and right hippocampus. Thus, it can be inferred that the metabolic level of left and right mPFC should be consistent. In addition, the study found that PV-expressing neurons infected by rAAV virus mainly existed in the MPFC, and also slightly exhibited in the the mediodorsal thalamus, striatum, cortex, etc., therefore, optogenetics should directly regulate PV neurons of the MPFC but affect the whole working memory neural circuit of hippocampus-mediodorsal thalamus-mPFC in rats with $\mathrm{VCl}$, however, its specific mechanism needs to be further clarified.

\section{Conclusion}

To sum up, in vivo ${ }^{1} \mathrm{H}-\mathrm{MRS}$ is a promising tool for longitudinal tracing of neurochemicals and metabolism in the brain. The study revealed that neurochemical metabolism was impaired in working memory neural circuit of hippocampus-mediodorsal thalamus-mPFC as early as $1 \sim 4$ weeks after chronic cerebral ischemia and demonstrated that optogenetics modulation of PV neurons in the MPFC can improve the pathological changes and enhance working memory, which provides a theoretical and experimental basis for early diagnosis and treatment of $\mathrm{VCl}$.

\section{Abbreviations}

VCl: vascular cognitive impairment

${ }^{1} \mathrm{H}$-MRS: hydrogen proton magnetic resonance spectroscopy

NAA: N-acetylaspartate

mPFC: medial prefrontal cortex

GABA: gamma-aminobutyric acid

ml: myo-inositol

Glu: glutamate 
Cho: choline

PV: parvalbumin

VD: Vascular dementia

Lac: lactic acid

Gln: glutamine

2-VO: bilateral common carotid arteries

OptoStim: optogenetics stimulation group

Sham-OptoStim: sham-optogenetics stimulation group

ChR2: channelrhodopsin-2

NpHR: halorhodopsin

3D TOF: 3D time-of-fight

TR: Repetition Time

TE: Echo Time

FOV: Field of view

ROI: regions of interest

Cr: creatine

ppm: part per million

NOR: Novel object recognition test

GAD67: glutamate decarboxylase 67

NMDAR: N-methyl-D-aspartic acid receptor

ANOVA: analysis of variance

\section{Declarations}

\section{Ethical Approval and Consent to participate}


Experimental animals are raised by the Experimental Animal Center of Fujian University of Traditional Chinese Medicine [license number: SYXK (Min) 2019-0007]. All experimental procedures are strictly in accordance with the International Ethical Guidelines and the National Institutes of Health Guide for the Care and Use of Laboratory Animals, and were approved by the Ethics Committee of Fujian University of Traditional Chinese Medicine.

\section{Consent for publication}

All authors have read the manuscript and indicated consent for publication.

\section{Availability of supporting data}

The data generated during this study is included in this published article [and its supplementary file: Figure S1-S18, Table S1].

\section{Competing interests}

The authors declare that they have no conflict of interest.

\section{Funding}

This study was supported by grants from the National Natural Science Foundation of China (Grant No. 81873355) and the Science and technology platform construction project of Fujian science and Technology Department (Grant No.2018Y2002).

\section{Authors' contributions}

W.L.L., H.W.L. and T.T.J. contributed to the experimental design. H.W.L., S.X.L. and M.G.Y. contributed to the ${ }^{1} \mathrm{H}-\mathrm{MRS}$ scans and data analysis. T.T.J. and H.W.L. contributed to the optogenetics modulation of PV neurons for VCI. W.L.L., H.W.L., T.T.J., L.W.C., Y.L.D. and W.W.J. contributed to most of the data collection, discussion, and interpretation. All authors read and approved the final manuscript.

\section{Acknowledgements}

We thank Prof. Shan Baoci at Institute of High Energy Physics, Chinese Academy of Sciences for the help in the analysis of hydrogen proton magnetic resonance spectroscopy.

\section{References}

Auger, M.L., Floresco, S.B., 2014. Prefrontal cortical GABA modulation of spatial reference and working memory. Int J Neuropsychopharmacol 18.

Battaglioli, G., Liu, H., Martin, D.L., 2003. Kinetic differences between the isoforms of glutamate decarboxylase: implications for the regulation of GABA synthesis. J NEUROCHEM 86, 879-887. 
Bella, R., Cantone, M., Lanza, G., Ferri, R., Vinciguerra, L., Puglisi, V., Pennisi, M., Ricceri, R., Di Lazzaro, V., Pennisi, G., 2016. Cholinergic circuitry functioning in patients with vascular cognitive impairment-no dementia. BRAIN STIMUL 9, 225-233.

Bertolino, A., Frye, M., Callicott, J.H., Mattay, V.S., Rakow, R., Shelton-Repella, J., Post, R., Weinberger, D.R., 2003. Neuronal pathology in the hippocampal area of patients with bipolar disorder: a study with proton magnetic resonance spectroscopic imaging. Biol Psychiatry 53, 906-913.

Bezalel, V., Paz, R., Tal, A., 2019. Inhibitory and excitatory mechanisms in the human cingulate-cortex support reinforcement learning: A functional Proton Magnetic Resonance Spectroscopy study. NEUROIMAGE 184, 25-35.

Bolkan, S.S., Stujenske, J.M., Parnaudeau, S., Spellman, T.J., Rauffenbart, C., Abbas, A.I., Harris, A.Z., Gordon, J.A., Kellendonk, C., 2017. Thalamic projections sustain prefrontal activity during working memory maintenance. NAT NEUROSCI 20, 987-996.

Bruhn, H., Frahm, J., Gyngell, M.L., Merboldt, K.D., Hanicke, W., Sauter, R., 1989. Cerebral metabolism in man after acute stroke: new observations using localized proton NMR spectroscopy. MAGN RESON MED $9,126-131$.

Buzsáki, G., Draguhn, A., 2004. Neuronal oscillations in cortical networks. SCIENCE 304, 1926-1929.

Cardin, J.A., Carlén, M., Meletis, K., Knoblich, U., Zhang, F., Deisseroth, K., Tsai, L.H., Moore, C.I., 2009. Driving fast-spiking cells induces gamma rhythm and controls sensory responses. NATURE 459, 663-667.

Chen, S.Q., Cai, Q., Shen, Y.Y., Xu, C.X., Zhou, H., Zhao, Z., 2016. Hydrogen Proton Magnetic Resonance Spectroscopy in Multidomain Amnestic Mild Cognitive Impairment and Vascular Cognitive Impairment Without Dementia. Am J Alzheimers Dis Other Demen 31, 422-429.

Deisseroth, K., 2011. Optogenetics. NAT METHODS 8, 26-29.

Deisseroth, K., Feng, G., Majewska, A.K., Miesenböck, G., Ting, A., Schnitzer, M.J., 2006. Next-generation optical technologies for illuminating genetically targeted brain circuits. J NEUROSCI 26, 10380-10386.

Demougeot, C., Marie, C., Giroud, M., Beley, A., 2004. N-acetylaspartate: a literature review of animal research on brain ischaemia. J NEUROCHEM 90, 776-783.

D'Esposito, M., Postle, B.R., 2015. The cognitive neuroscience of working memory. ANNU REV PSYCHOL $66,115-142$.

Dichgans, M., Leys, D., 2017. Vascular Cognitive Impairment. CIRC RES 120, 573-591.

Dolleman-van, D.W.M., Griffin, A.L., Ito, H.T., Shapiro, M.L., Witter, M.P., Vertes, R.P., Allen, T.A., 2019. The nucleus reuniens of the thalamus sits at the nexus of a hippocampus and medial prefrontal cortex circuit 
enabling memory and behavior. Learn Mem 26, 191-205.

Eichenbaum, H., 2017. Prefrontal-hippocampal interactions in episodic memory. NAT REV NEUROSCI 18, 547-558.

Esclapez, M., Tillakaratne, N.J., Kaufman, D.L., Tobin, A.J., Houser, C.R., 1994. Comparative localization of two forms of glutamic acid decarboxylase and their mRNAs in rat brain supports the concept of functional differences between the forms. J NEUROSCI 14, 1834-1855.

Farkas, E., Luiten, P.G., Bari, F., 2007. Permanent, bilateral common carotid artery occlusion in the rat: a model for chronic cerebral hypoperfusion-related neurodegenerative diseases. Brain Res Rev 54, 162-180.

Ferguson, B.R., Gao, W.J., 2018a. PV Interneurons: Critical Regulators of E/I Balance for Prefrontal CortexDependent Behavior and Psychiatric Disorders. Front Neural Circuits 12, 37.

Ferguson, B.R., Gao, W.J., 2018b. PV Interneurons: Critical Regulators of E/I Balance for Prefrontal CortexDependent Behavior and Psychiatric Disorders. Front Neural Circuits 12, 37.

Finn, E.S., Huber, L., Jangraw, D.C., Molfese, P.J., Bandettini, P.A., 2019. Layer-dependent activity in human prefrontal cortex during working memory. NAT NEUROSCI 22, 1687-1695.

Galisova, A., Baciak, L., Jozefovicova, M., Just, K.I., Kebis, A., Ambrusova, K., Dubovicky, M., Estera, C., Sadlonova, I., Kronnerwetter, C., Berg, A., Krssak, M., Kasparova, S., 2014. Pathophysiological rat model of vascular dementia: magnetic resonance spectroscopy, microimaging and behavioral study. BRAIN RES $1568,10-20$.

Gasparovic, C., Prestopnik, J., Thompson, J., Taheri, S., Huisa, B., Schrader, R., Adair, J.C., Rosenberg, G.A., 2013. 1 H-MR spectroscopy metabolite levels correlate with executive function in vascular cognitive impairment. J Neurol Neurosurg Psychiatry 84, 715-721.

Glanville, N.T., Byers, D.M., Cook, H.W., Spence, M.W., Palmer, F.B., 1989. Differences in the metabolism of inositol and phosphoinositides by cultured cells of neuronal and glial origin. Biochim Biophys Acta 1004, 169-179.

Goldman-Rakic, P.S., 1995. Cellular basis of working memory. NEURON 14, 477-485.

Goodman, R.A., Lochner, K.A., Thambisetty, M., Wingo, T.S., Posner, S.F., Ling, S.M., 2017. Prevalence of dementia subtypes in United States Medicare fee-for-service beneficiaries, 2011-2013. ALZHEIMERS DEMENT 13, 28-37.

Gorelick, P.B., Scuteri, A., Black, S.E., Decarli, C., Greenberg, S.M., ladecola, C., Launer, L.J., Laurent, S., Lopez, O.L., Nyenhuis, D., Petersen, R.C., Schneider, J.A., Tzourio, C., Arnett, D.K., Bennett, D.A., Chui, H.C., Higashida, R.T., Lindquist, R., Nilsson, P.M., Roman, G.C., Sellke, F.W., Seshadri, S., 2011. Vascular 
contributions to cognitive impairment and dementia: a statement for healthcare professionals from the american heart association/american stroke association. STROKE 42, 2672-2713.

Gurol, M.E., 2016. Molecular Neuroimaging in Vascular Cognitive Impairment. STROKE 47, 1146-1152.

Han, Q.Y., Zhang, H., Zhang, X., He, D.S., Wang, S.W., Cao, X., Dai, Y.T., Xu, Y., Han, L.J., 2019. dl-3-nbutylphthalide preserves white matter integrity and alleviates cognitive impairment in mice with chronic cerebral hypoperfusion. CNS NEUROSCI THER 25, 1042-1053.

He, J., Zhao, C., Liu, W., Huang, J., Liang, S., Chen, L., Tao, J., 2018. Neurochemical changes in the hippocampus and prefrontal cortex associated with electroacupuncture for learning and memory impairment. INT J MOL MED 41, 709-716.

Hu, H., Gan, J., Jonas, P., 2014. Interneurons. Fast-spiking, parvalbumin $\rrbracket$ GABAergic interneurons: from cellular design to microcircuit function. SCIENCE 345, 1255263.

Hu, Z.L., Xia, H.H., Yang, Y.J., Zheng, H., Zhao, L.C., Chen, Y.C., Zhuge, Q.C., Xia, N.Z., Gao, H.C., Chen, W.J., 2018. Metabolic alterations in the rat cerebellum following acute middle cerebral artery occlusion, as determined by $1 \mathrm{H}$ NMR spectroscopy. MOL MED REP 17, 531-541.

Huang, M., Kwon, S., Rajagopal, L., He, W., Meltzer, H.Y., 2018. 5-HT1A parital agonism and 5-HT7 antagonism restore episodic memory in subchronic phencyclidine-treated mice: role of brain glutamate, dopamine, acetylcholine and GABA. Psychopharmacology (Berl) 235, 2795-2808.

Hwang, S., Ham, S., Lee, S.E., Lee, Y., Lee, G.H., 2018. Hypoxia regulates the level of glutamic acid decarboxylase enzymes and interrupts inhibitory synapse stability in primary cultured neurons. NEUROTOXICOLOGY 65, 221-230.

ladecola, C., Duering, M., Hachinski, V., Joutel, A., Pendlebury, S.T., Schneider, J.A., Dichgans, M., 2019. Vascular Cognitive Impairment and Dementia: JACC Scientific Expert Panel. J AM COLL CARDIOL 73, 3326-3344.

Igarashi, H., Kwee, I.L., Nakada, T., Katayama, Y., Terashi, A., 2001. $1 \mathrm{H}$ magnetic resonance spectroscopic imaging of permanent focal cerebral ischemia in rat: longitudinal metabolic changes in ischemic core and rim. BRAIN RES 907, 208-221.

Izaki, Y., Takita, M., Akema, T., 2008. Specific role of the posterior dorsal hippocampus-prefrontal cortex in short-term working memory. EUR J NEUROSCI 27, 3029-3034.

Jayaweera, H.K., Lagopoulos, J., Duffy, S.L., Lewis, S.J., Hermens, D.F., Norrie, L., Hickie, I.B., Naismith, S.L., 2015. Spectroscopic markers of memory impairment, symptom severity and age of onset in older people with lifetime depression: Discrete roles of $\mathrm{N}$-acetyl aspartate and glutamate. J Affect Disord 183, 31-38. 
Jellinger, K.A., 2008. Morphologic diagnosis of "vascular dementia" - a critical update. J NEUROL SCI 270, $1-12$.

Jin, X., Li, T., Zhang, L., Ma, J., Yu, L., Li, C., Niu, L., 2017. Environmental Enrichment Improves Spatial Learning and Memory in Vascular Dementia Rats with Activation of Wnt/beta-Catenin Signal Pathway. Med Sci Monit 23, 207-215.

Jones, R.S., Waldman, A.D., 2004. 1H-MRS evaluation of metabolism in Alzheimer's disease and vascular dementia. NEUROL RES 26, 488-495.

Jun, C., Choi, Y., Lim, S.M., Bae, S., Hong, Y.S., Kim, J.E., Lyoo, I.K., 2014. Disturbance of the glutamatergic system in mood disorders. EXP NEUROBIOL 23, 28-35.

Kalaria, R.N., 2016. Neuropathological diagnosis of vascular cognitive impairment and vascular dementia with implications for Alzheimer's disease. ACTA NEUROPATHOL 131, 659-685.

Kertesz, A., Clydesdale, S., 1994. Neuropsychological deficits in vascular dementia vs Alzheimer's disease. Frontal lobe deficits prominent in vascular dementia. Arch Neurol 51, 1226-1231.

Klein, J., 2000. Membrane breakdown in acute and chronic neurodegeneration: focus on cholinecontaining phospholipids. J Neural Transm (Vienna) 107, 1027-1063.

Krahe, J., Binkofski, F., Schulz, J.B., Reetz, K., Romanzetti, S., 2020. Neurochemical profiles in hereditary ataxias: A meta-analysis of Magnetic Resonance Spectroscopy studies. Neurosci Biobehav Rev 108, 854865.

Kupferschmidt, D.A., Gordon, J.A., 2018. The dynamics of disordered dialogue: Prefrontal, hippocampal and thalamic miscommunication underlying working memory deficits in schizophrenia. Brain Neurosci Adv 2.

Kuroda, M., Yokofujita, J., Murakami, K., 1998. An ultrastructural study of the neural circuit between the prefrontal cortex and the mediodorsal nucleus of the thalamus. PROG NEUROBIOL 54, 417-458.

Lazarus, M.S., Krishnan, K., Huang, Z.J., 2015. GAD67 deficiency in parvalbumin interneurons produces deficits in inhibitory transmission and network disinhibition in mouse prefrontal cortex. CEREB CORTEX $25,1290-1296$.

Li, Q., Yang, C., Zhang, X., Yang, Z., Zhang, T., 2019. Arginine vasopressin attenuates dysfunction of hippocampal theta and gamma oscillations in chronic cerebral hypoperfusion via V1a receptor. BRAIN RES BULL 153, 84-92.

Liang, S., Zhang, J., Zhang, Q., Li, L., Zhang, Y., Jin, T., Zhang, B., He, X., Chen, L., Tao, J., Li, Z., Liu, W., Chen, L., 2020. Longitudinal tracing of white matter integrity on diffusion tensor imaging in the chronic cerebral ischemia and acute cerebral ischemia. BRAIN RES BULL 154, 135-141. 
Liu, H., Wang, X., 2016. Correlation of iron deposition and change of gliocyte metabolism in the basal ganglia region evaluated using magnetic resonance imaging techniques: an in vivo study. ARCH MED SCl 12, 163-171.

Marsman, A., Mandl, R., Klomp, D., Cahn, W., Kahn, R.S., Luijten, P.R., Hulshoff, P.H., 2017. Intelligence and Brain Efficiency: Investigating the Association between Working Memory Performance, Glutamate, and GABA. FRONT PSYCHIATRY 8, 154.

Mattfeld, A.T., Stark, C.E., 2015. Functional contributions and interactions between the human hippocampus and subregions of the striatum during arbitrary associative learning and memory. HIPPOCAMPUS 25, 900-911.

McGuinness, B., Barrett, S.L., Craig, D., Lawson, J., Passmore, A.P., 2010. Executive functioning in Alzheimer's disease and vascular dementia. Int J Geriatr Psychiatry 25, 562-568.

Miller, E.K., Cohen, J.D., 2001. An integrative theory of prefrontal cortex function. ANNU REV NEUROSCI 24, 167-202.

Miller, R., Francoeur, M.J., Gibson, B.M., Mair, R.G., 2017. Mediodorsal Thalamic Neurons Mirror the Activity of Medial Prefrontal Neurons Responding to Movement and Reinforcement during a Dynamic DNMTP Task. eNeuro 4.

O'Brien, J.T., Thomas, A., 2015. Vascular dementia. LANCET 386, 1698-1706.

O'Neill, P.K., Gordon, J.A., Sigurdsson, T., 2013. Theta oscillations in the medial prefrontal cortex are modulated by spatial working memory and synchronize with the hippocampus through its ventral subregion. J NEUROSCI 33, 14211-14224.

Park, K., Lee, J., Jang, H.J., Richards, B.A., Kohl, M.M., Kwag, J., 2020. Optogenetic activation of parvalbumin and somatostatin interneurons selectively restores theta-nested gamma oscillations and oscillation-induced spike timing-dependent long-term potentiation impaired by amyloid $\beta$ oligomers. BMC BIOL 18, 7 .

Parnaudeau, S., Bolkan, S.S., Kellendonk, C., 2018. The Mediodorsal Thalamus: An Essential Partner of the Prefrontal Cortex for Cognition. Biol Psychiatry 83, 648-656.

Parnaudeau, S., O'Neill, P.K., Bolkan, S.S., Ward, R.D., Abbas, A.I., Roth, B.L., Balsam, P.D., Gordon, J.A., Kellendonk, C., 2013. Inhibition of mediodorsal thalamus disrupts thalamofrontal connectivity and cognition. NEURON 77, 1151-1162.

Preston, A.R., Eichenbaum, H., 2013. Interplay of hippocampus and prefrontal cortex in memory. CURR BIOL 23, R764-R773.

Rajani, V., Sengar, A.S., Salter, M.W., 2020. Tripartite signalling by NMDA receptors. MOL BRAIN 13, 23. 
Rutten, K., Reneerkens, O.A., Hamers, H., Sik, A., McGregor, I.S., Prickaerts, J., Blokland, A., 2008. Automated scoring of novel object recognition in rats. J Neurosci Methods 171, 72-77.

Schmitt, L.I., Wimmer, R.D., Nakajima, M., Happ, M., Mofakham, S., Halassa, M.M., 2017. Thalamic amplification of cortical connectivity sustains attentional control. NATURE 545, 219-223.

Selimbeyoglu, A., Kim, C.K., Inoue, M., Lee, S.Y., Hong, A., Kauvar, I., Ramakrishnan, C., Fenno, L.E., Davidson, T.J., Wright, M., Deisseroth, K., 2017. Modulation of prefrontal cortex excitation/inhibition balance rescues social behavior in CNTNAP2-deficient mice. SCI TRANSL MED 9.

Sohal, V.S., Zhang, F., Yizhar, O., Deisseroth, K., 2009. Parvalbumin neurons and gamma rhythms enhance cortical circuit performance. NATURE 459, 698-702.

Sohn, E., Kim, Y.J., Lim, H.S., Kim, B.Y., Jeong, S.J., 2019. Hwangryunhaedok-Tang Exerts Neuropreventive Effect on Memory Impairment by Reducing Cholinergic System Dysfunction and Inflammatory Response in a Vascular Dementia Rat Model. MOLECULES 24.

Soria, G., Tudela, R., Márquez-Martín, A., Camón, L., Batalle, D., Muñoz-Moreno, E., Eixarch, E., Puig, J., Pedraza, S., Vila, E., Prats-Galino, A., Planas, A.M., 2013. The ins and outs of the BCCAo model for chronic hypoperfusion: a multimodal and longitudinal MRI approach. PLOS ONE 8, e74631.

Stoiljkovic, M., Leventhal, L., Chen, A., Chen, T., Driscoll, R., Flood, D., Hodgdon, H., Hurst, R., Nagy, D., Piser, T., Tang, C., Townsend, M., Tu, Z., Bertrand, D., Koenig, G., Hajos, M., 2015. Concentration-response relationship of the alpha7 nicotinic acetylcholine receptor agonist FRM-17874 across multiple in vitro and in vivo assays. BIOCHEM PHARMACOL 97, 576-589.

Tamura, M., Spellman, T.J., Rosen, A.M., Gogos, J.A., Gordon, J.A., 2017. Hippocampal-prefrontal thetagamma coupling during performance of a spatial working memory task. NAT COMMUN 8, 2182.

Thielen, J.W., Hong, D., Rohani, R.S., Wiltfang, J., Fernandez, G., Norris, D.G., Tendolkar, I., 2018. The increase in medial prefrontal glutamate/glutamine concentration during memory encoding is associated with better memory performance and stronger functional connectivity in the human medial prefrontalthalamus-hippocampus network. HUM BRAIN MAPP 39, 2381-2390.

Tiwari, V., Ambadipudi, S., Patel, A.B., 2013. Glutamatergic and GABAergic TCA cycle and neurotransmitter cycling fluxes in different regions of mouse brain. J Cereb Blood Flow Metab 33, 1523-1531.

Tomaszewicz, M., Rossner, S., Schliebs, R., Cwikowska, J., Szutowicz, A., 2003. Changes in cortical acetylCoA metabolism after selective basal forebrain cholinergic degeneration by 192lgG-saporin. J NEUROCHEM 87, 318-324.

Tomimoto, H., Ihara, M., Wakita, H., Ohtani, R., Lin, J.X., Akiguchi, I., Kinoshita, M., Shibasaki, H., 2003. Chronic cerebral hypoperfusion induces white matter lesions and loss of oligodendroglia with DNA fragmentation in the rat. ACTA NEUROPATHOL 106, 527-534. 
van der Flier, W.M., Skoog, I., Schneider, J.A., Pantoni, L., Mok, V., Chen, C., Scheltens, P., 2018. Vascular cognitive impairment. NAT REV DIS PRIMERS 4, 18003.

Varela, C., Kumar, S., Yang, J.Y., Wilson, M.A., 2014. Anatomical substrates for direct interactions between hippocampus, medial prefrontal cortex, and the thalamic nucleus reuniens. BRAIN STRUCT FUNCT 219, 911-929.

Venkat, P., Chopp, M., Chen, J., 2015. Models and mechanisms of vascular dementia. EXP NEUROL 272, 97-108.

Wang, J., Zhang, H.Y., Tang, X.C., 2009. Cholinergic deficiency involved in vascular dementia: possible mechanism and strategy of treatment. ACTA PHARMACOL SIN 30, 879-888.

Wang, K.W., Ye, X.L., Huang, T., Yang, X.F., Zou, L.Y., 2019. Optogenetics-induced activation of glutamate receptors improves memory function in mice with Alzheimer's disease. NEURAL REGEN RES 14, 21472155.

Wang, X., Lin, F., Gao, Y., Lei, H., 2015. Bilateral common carotid artery occlusion induced brain lesions in rats: A longitudinal diffusion tensor imaging study. MAGN RESON IMAGING 33, 551-558.

Xi, G., Hui, J., Zhang, Z., Liu, S., Zhang, X., Teng, G., Chan, K.C., Wu, E.X., Nie, B., Shan, B., Li, L., Reynolds, G.P., 2011. Learning and memory alterations are associated with hippocampal $\mathrm{N}$-acetylaspartate in a rat model of depression as measured by 1H-MRS. PLOS ONE 6, e28686.

Xu, X., Li, Z., Yang, Z., Zhang, T., 2012. Decrease of synaptic plasticity associated with alteration of information flow in a rat model of vascular dementia. NEUROSCIENCE 206, 136-143.

Yang, M., Wang, S., Hao, F., Li, Y., Tang, H., Shi, X., 2012. NMR analysis of the rat neurochemical changes induced by middle cerebral artery occlusion. TALANTA 88, 136-144.

Yang, T., Sun, Y., Lu, Z., Leak, R.K., Zhang, F., 2017. The impact of cerebrovascular aging on vascular cognitive impairment and dementia. AGEING RES REV 34, 15-29.

Zhou, I.Y., Chan, R.W., Ho, L.C., Wu, E.X., 2013. Longitudinal metabolic changes in the hippocampus and thalamus of the maternal brain revealed by proton magnetic resonance spectroscopy. NEUROSCI LETT $553,170-175$.

Zhou, I.Y., Ding, A.Y., Li, Q., McAlonan, G.M., Wu, E.X., 2012. Magnetic resonance spectroscopy reveals Nacetylaspartate reduction in hippocampus and cingulate cortex after fear conditioning. Psychiatry Res 204, 178-183.

\section{Figures}


A

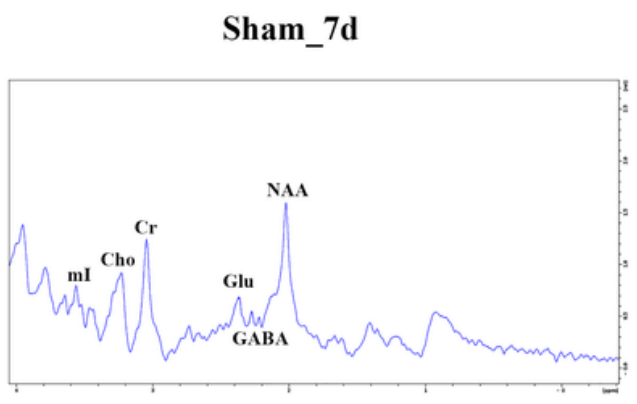

C

Sham_14d

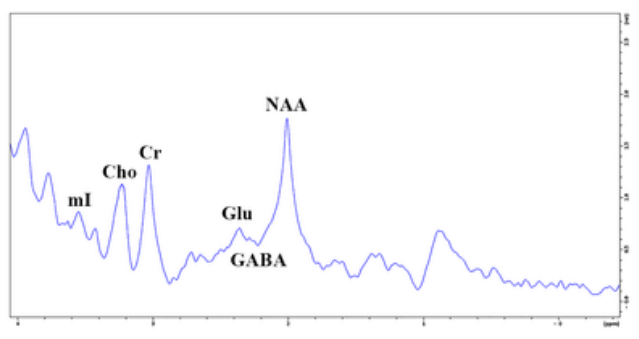

$\mathbf{E}$

Sham_28d

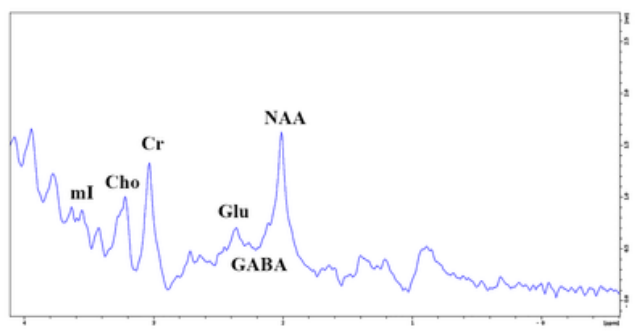

B

Right Hippocampus
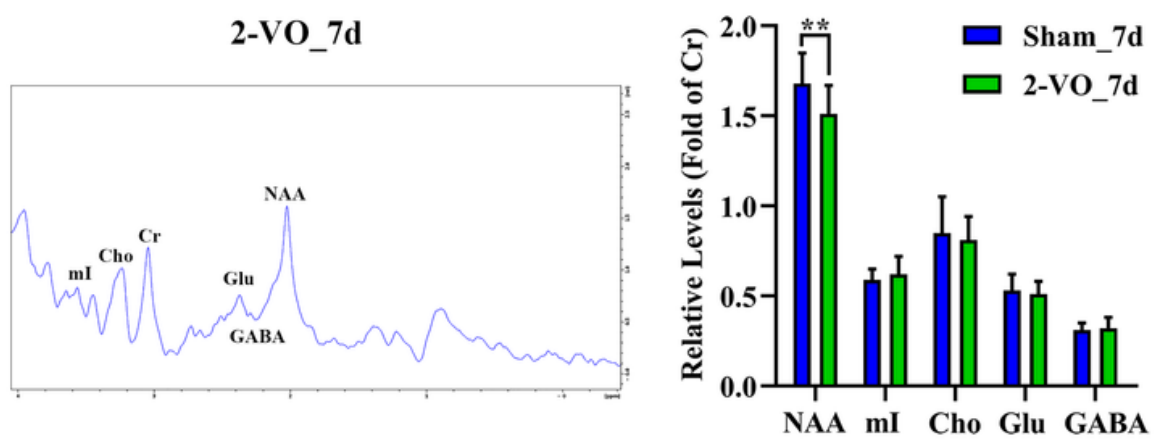

D Right Hippocampus

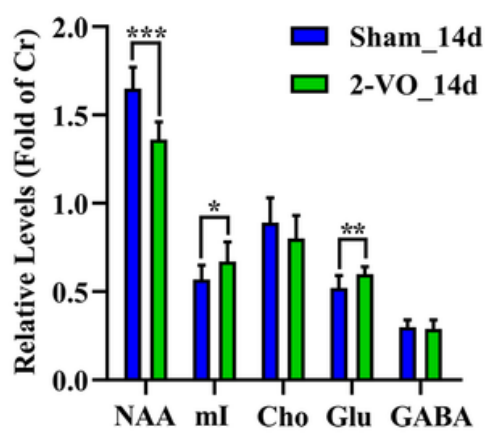

F Right Hippocampus

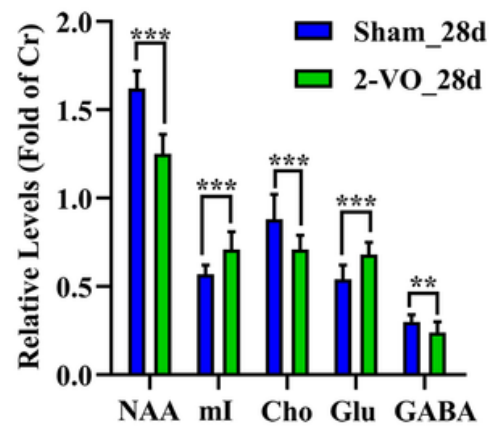

Figure 1

The time course of neurochemicals and metabolic changes in the right hippocampus following chronic cerebral ischemia. (A, C, E) Representative 1H-MRS graphs both the Sham group and the 2-VO group at 7 days, 14 days and 28 days after chronic cerebral ischemia, respectively. (B, D, F) Statistical results of neurochemicals (NAA, ml, Cho, Glu and GABA) between the sham group $(n=17)$ and the 2-Vo group $(\mathrm{n}=14)$ at 7 days, 14 days and 28 days after chronic cerebral ischemia, respectively. Abbreviation: $1 \mathrm{H}-$ MRS, hydrogen proton magnetic resonance spectroscopy; 2-VO, bilateral common carotid artery occlusion; ml, myoinositol; Cho, choline; $\mathrm{Cr}$, creatine; Glu, glutamate; NAA, N-acetylaspartate, GABA, $Y^{-}$ aminobutyric acid. *, $\mathrm{P}<0.05, * \star, \mathrm{P}<0.01 ; * \star \star, P<0.001$. 
A

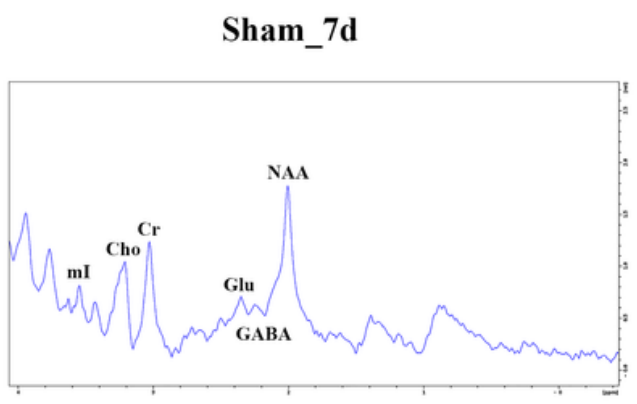

C

Sham_14d

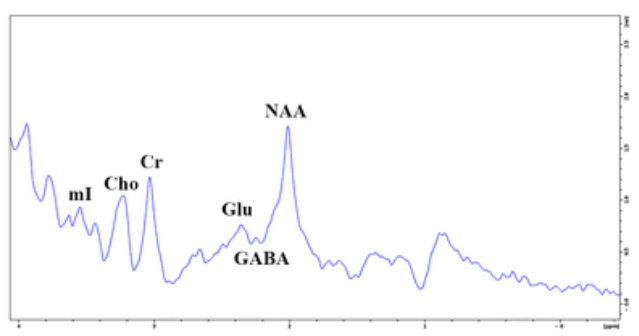

$\mathbf{E}$

Sham_28d

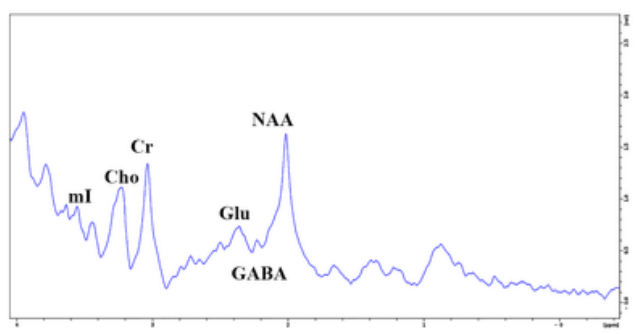

2-VO_7d

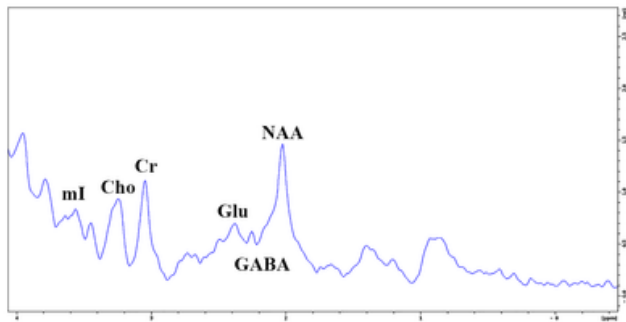

D

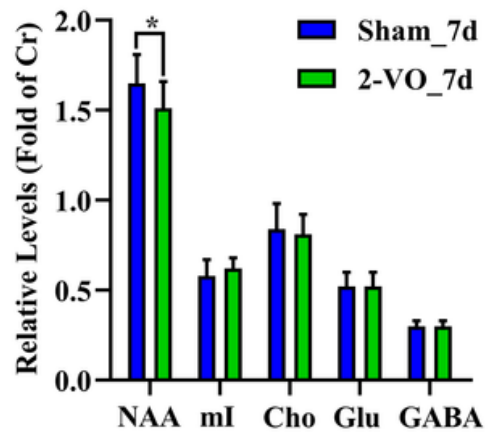

Deft Hippocampus

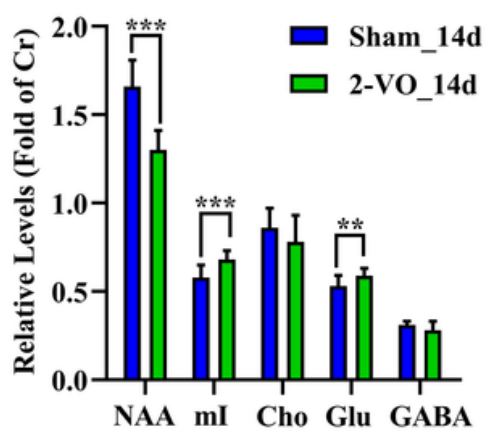

F Left Hippocampus

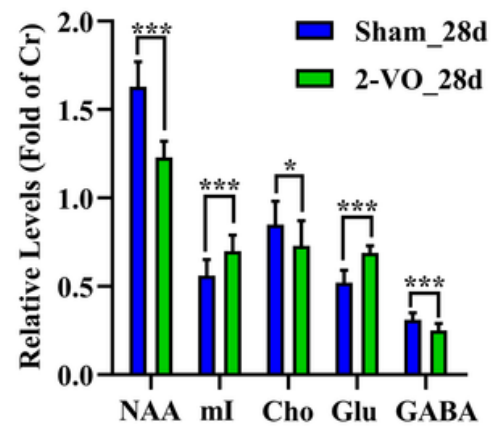

Figure 2

The time course of neurochemicals and metabolic changes in the left hippocampus following chronic cerebral ischemia. (A, C, E) Representative 1H-MRS graphs both the Sham group and the 2-VO group at 7 days, 14 days and 28 days after chronic cerebral ischemia, respectively. (B, D, F) Statistical results of neurochemicals (NAA, ml, Cho, Glu and GABA) between the sham group $(n=17)$ and the 2-Vo group $(\mathrm{n}=14)$ at 7 days, 14 days and 28 days after chronic cerebral ischemia, respectively. Abbreviation: $1 \mathrm{H}-$ MRS, hydrogen proton magnetic resonance spectroscopy; 2-VO, bilateral common carotid artery occlusion; ml, myoinositol; Cho, choline; $\mathrm{Cr}$, creatine; Glu, glutamate; NAA, N-acetylaspartate, GABA, $\mathrm{Y}^{-}$ aminobutyric acid. *, $\mathrm{P}<0.05$, **, $\mathrm{P}<0.01 ; * \star *, \mathrm{P}<0.001$. 
A

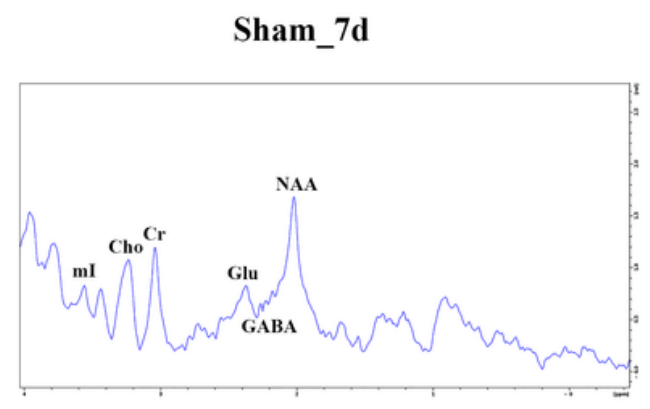

C

Sham_14d

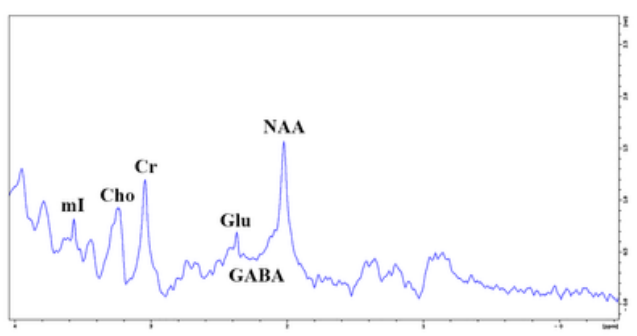

E

Sham_28d

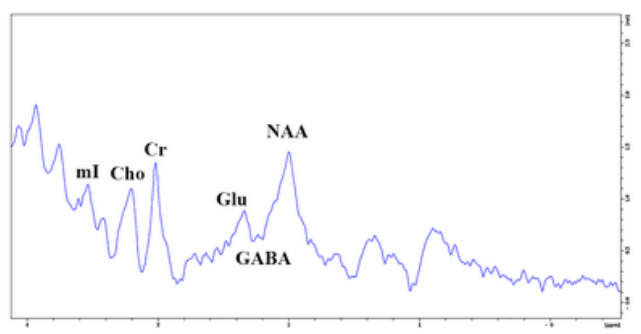

2-VO_7d

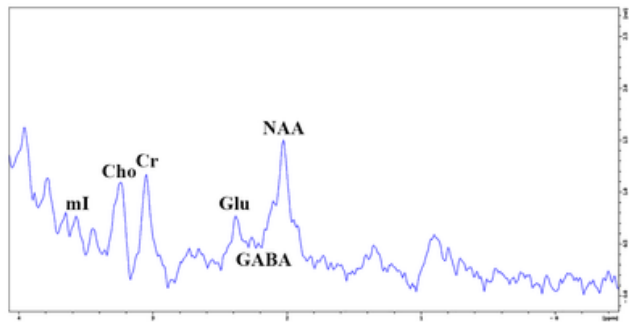

D

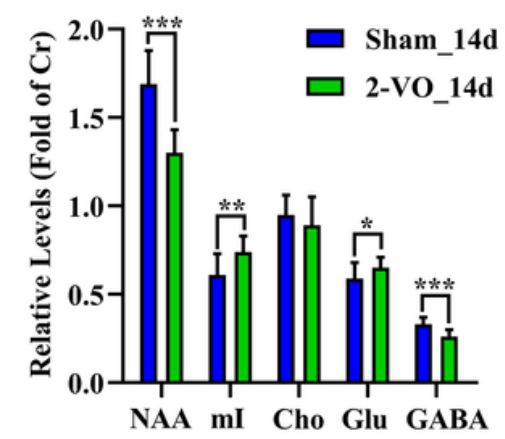

F

2-VO_28d

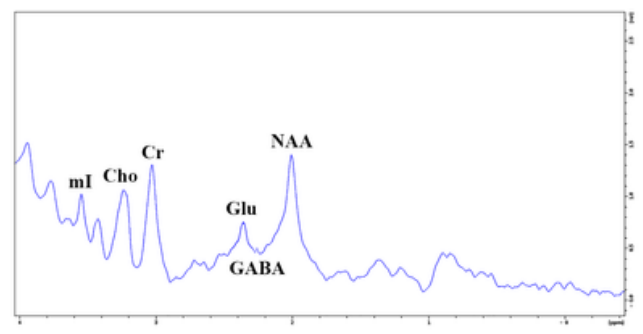

B

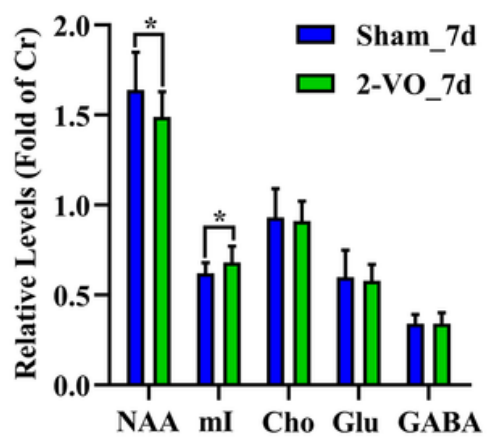

Right mPFC

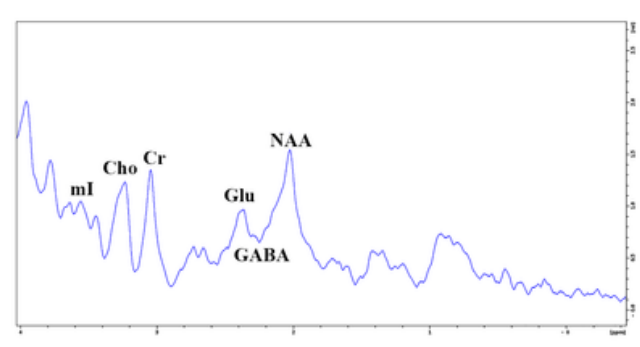

Right mPFC

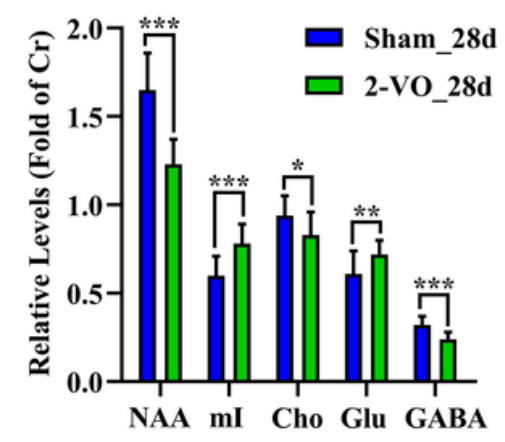

Figure 3

The time course of neurochemicals and metabolic changes in the right mPFC following chronic cerebral ischemia. (A, C, E) Representative 1H-MRS graphs both the Sham group and the 2-VO group at 7 days, 14 days and 28 days after chronic cerebral ischemia, respectively. (B, D, F) Statistical results of neurochemicals (NAA, ml, Cho, Glu and GABA) between the sham group $(n=17)$ and the 2-Vo group $(\mathrm{n}=14)$ at 7 days, 14 days and 28 days after chronic cerebral ischemia, respectively. Abbreviation: $1 \mathrm{H}-$ MRS, hydrogen proton magnetic resonance spectroscopy; mPFC, medial prefrontal cortex; 2-VO, bilateral common carotid artery occlusion; $\mathrm{ml}$, myoinositol; Cho, choline; $\mathrm{Cr}$, creatine; Glu, glutamate; NAA, Nacetylaspartate, GABA, $y$-aminobutyric acid. * ${ }^{*} \mathrm{P}<0.05$, **, $\mathrm{P}<0.01 ; * \star \star, P<0.001$. 
A

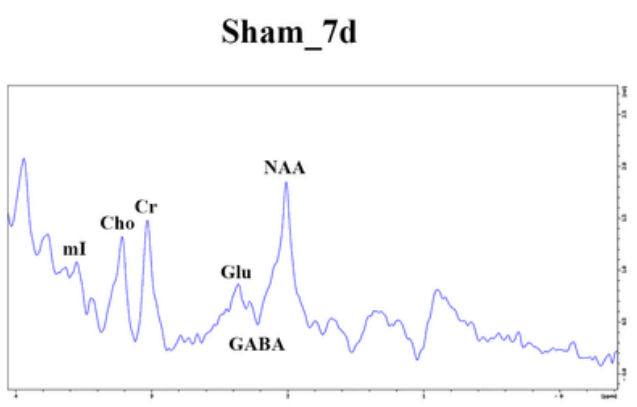

C

Sham_14d

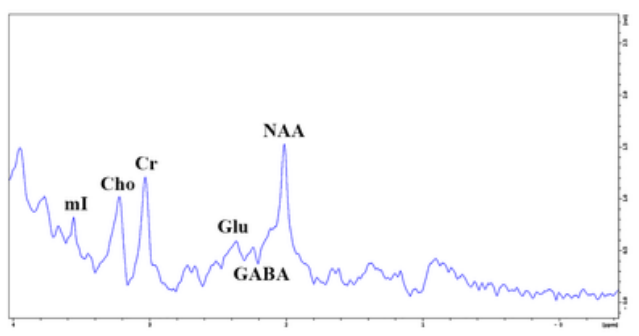

$\mathbf{E}$

Sham_28d

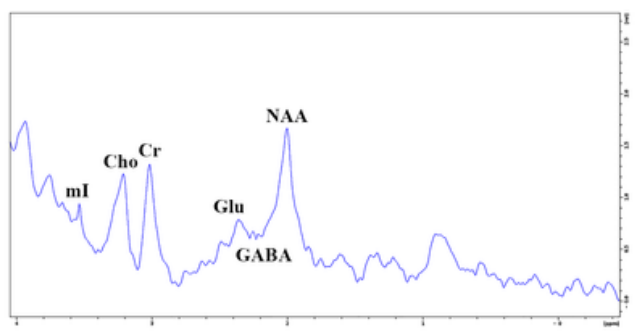

2-VO_7d

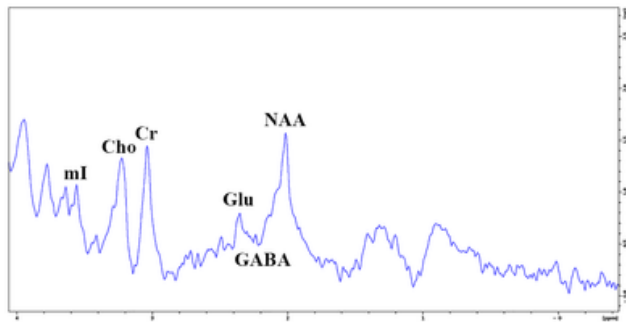

D Medidorsal Thalamus

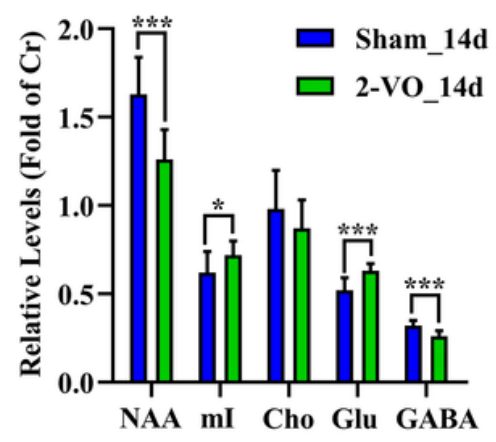

F Medidorsal Thalamus



Figure 4

The time course of neurochemicals and metabolic changes in the mediodorsal thalamus following chronic cerebral ischemia. (A, C, E) Representative 1H-MRS graphs both the Sham group and the 2-VO group at 7 days, 14 days and 28 days after chronic cerebral ischemia, respectively. (B, D, F) Statistical results of neurochemicals (NAA, $\mathrm{ml}$, Cho, Glu and GABA) between the sham group $(n=17)$ and the 2-VO group $(n=14)$ at 7 days, 14 days and 28 days after chronic cerebral ischemia, respectively. Abbreviation: $1 \mathrm{H}-\mathrm{MRS}$, hydrogen proton magnetic resonance spectroscopy; 2-VO, bilateral common carotid artery occlusion; ml, myoinositol; Cho, choline; $\mathrm{Cr}$, creatine; Glu, glutamate; NAA, N-acetylaspartate, GABA, $\mathrm{Y}^{-}$ aminobutyric acid. *, $\mathrm{P}<0.05$, **, $\mathrm{P}<0.01 ; \star \star \star, P<0.001$. 
A

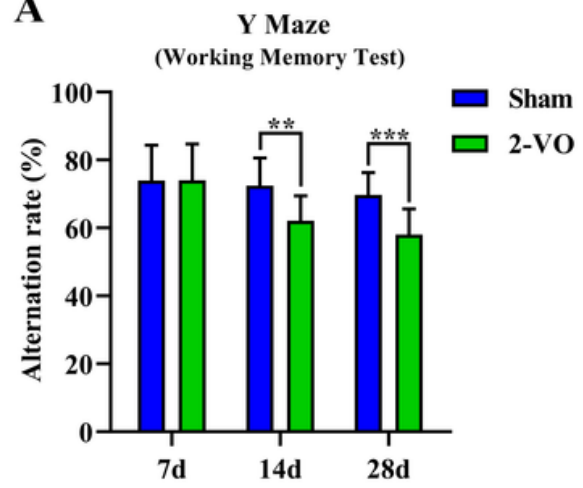

D

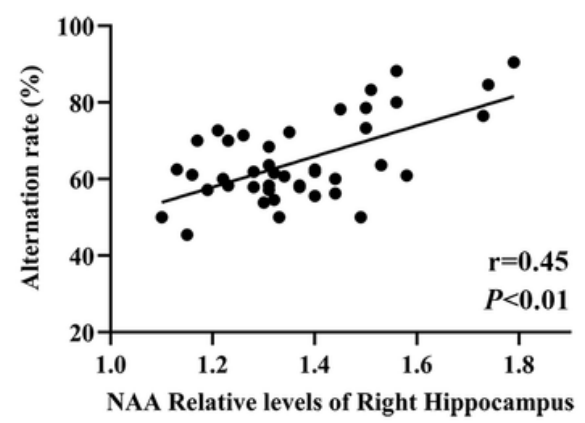

G

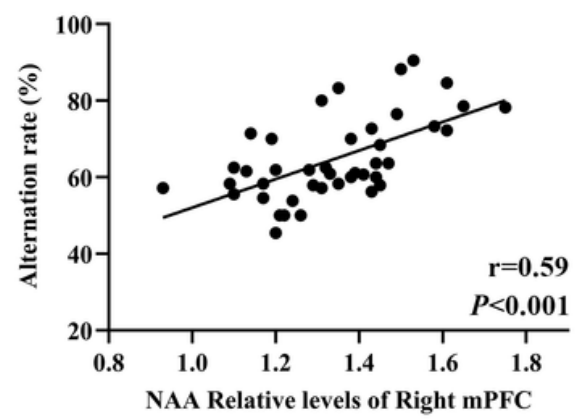

B

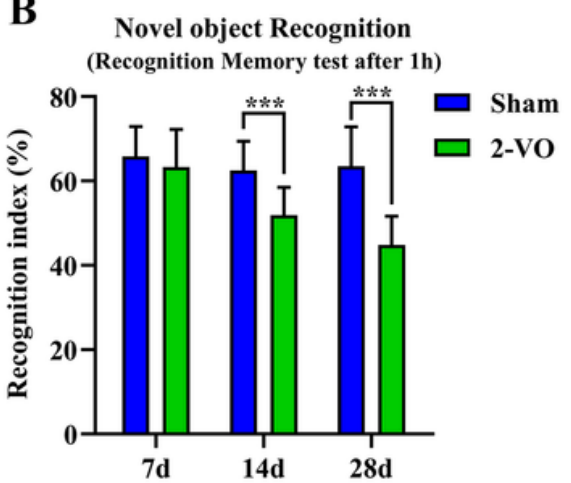

E

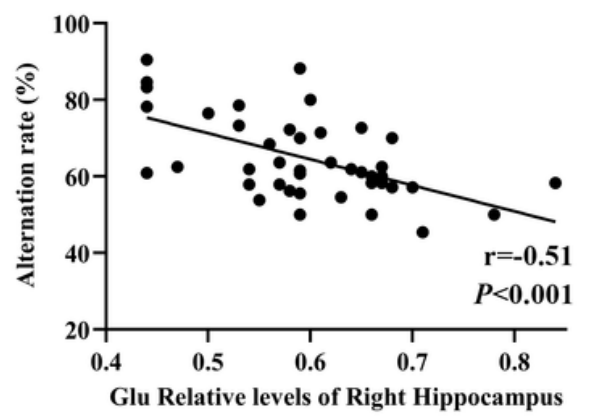

H



C

Novel object Recognition (Recognition Memory test after 24h)

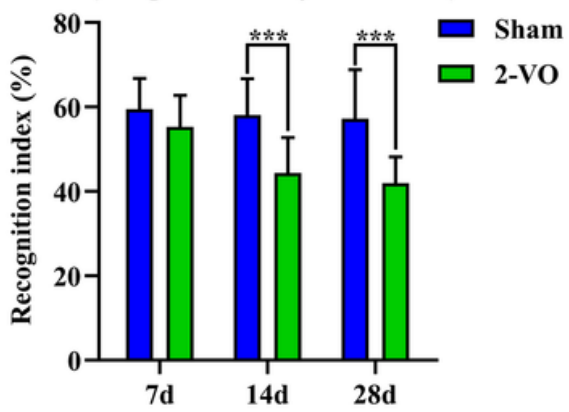

F

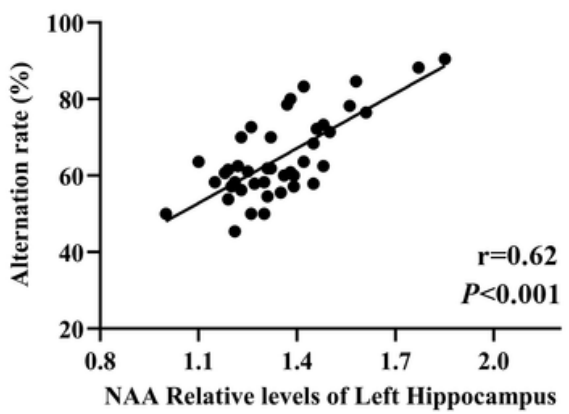

I

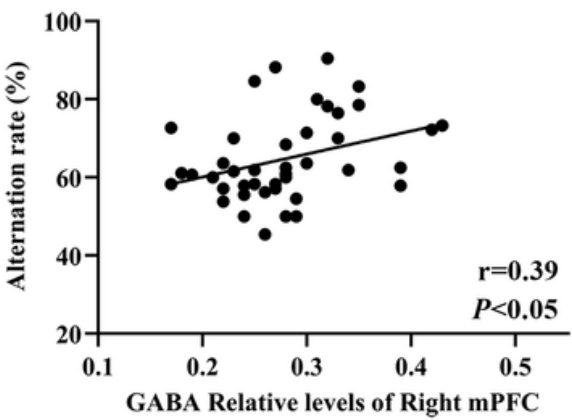

Figure 5

Working memory and object recognition memory were impaired and correlated with neurochemicals metabolic disorders after chronic cerebral ischemia. (A) The alternating rate in $\mathrm{Y}$ maze was tested for measuring working memory both the sham group $(n=17)$ and the 2 -VO group $(n=14)$ at 7 days, 14 days and 28 days after chronic cerebral ischemia, respectively. (B, C) The $1 \mathrm{~h}$ and $24 \mathrm{~h}$ recognition indices of NOR was tested both the sham group $(n=17)$ and the 2-VO group $(n=14)$ at 7 days, 14 days and 28 days after chronic cerebral ischemia, respectively. (D, F, G) Correlations between the NAA levels of the right hippocampus, left hippocampus, right mPFC and $Y$ maze alternation rate, respectively. $(E, H)$ Correlations between the Glu levels of the right hippocampus, right $\mathrm{MPFC}$ and $\mathrm{Y}$ maze alternation rate. (I) Correlation between GABA levels of the right $\mathrm{mPFC}$ and $\mathrm{Y}$ maze alternation rate. Abbreviation: $\mathrm{mPFC}$, medial prefrontal cortex; $\mathrm{Cr}$, creatine; Glu, glutamate; NAA, N-acetylaspartate; GABA, $\gamma$-aminobutyric acid; 2-VO, bilateral common carotid artery occlusion; NOR, novel object recognition. **, $\mathrm{P}<0.01$; ***, $\mathrm{P}<0.001$. 
A rAAV-Ef1a-DIO-hChR2-EYFP-WPRE-pA $473 \mathrm{~nm} \triangle \longrightarrow$

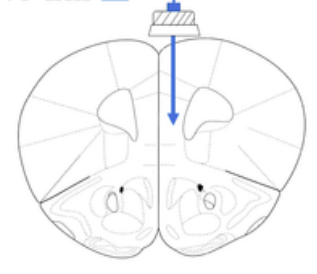

Right mPFC

C

Right mPFC: Sham-OptoStim

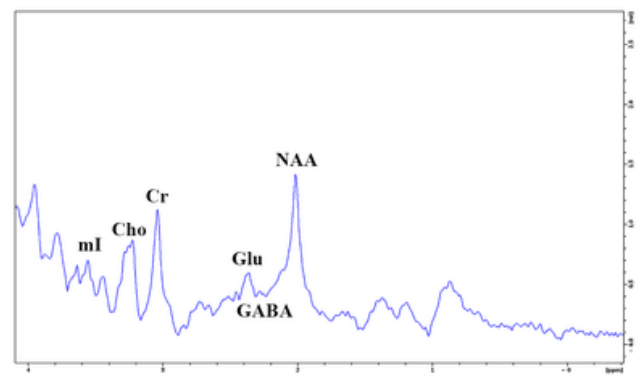

E

Right Hippocampus

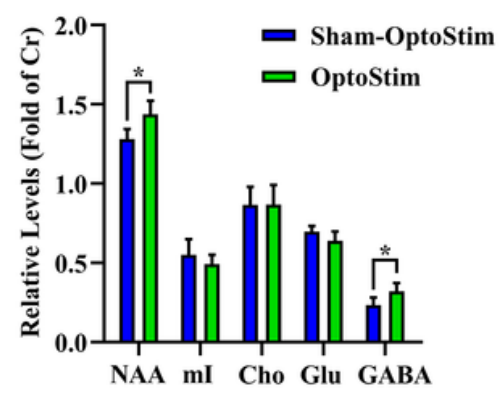

H

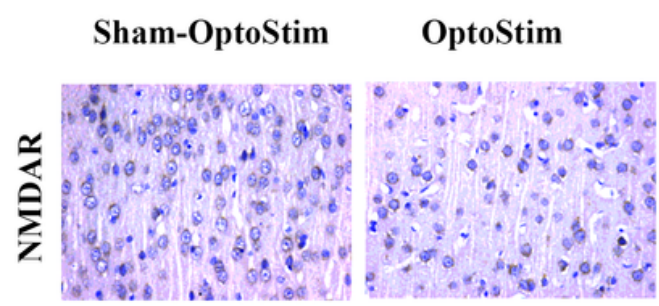

Sham-OptoStim


Right mPFC: OptoStim

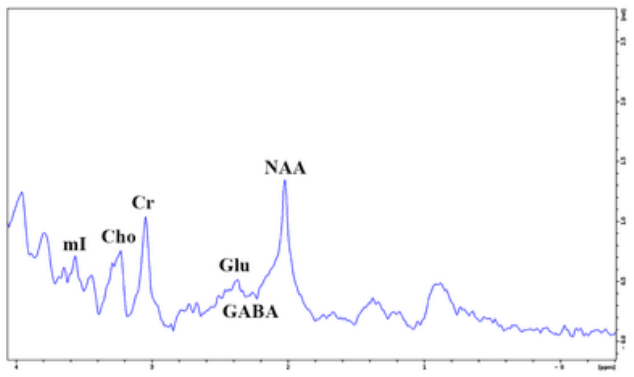

F
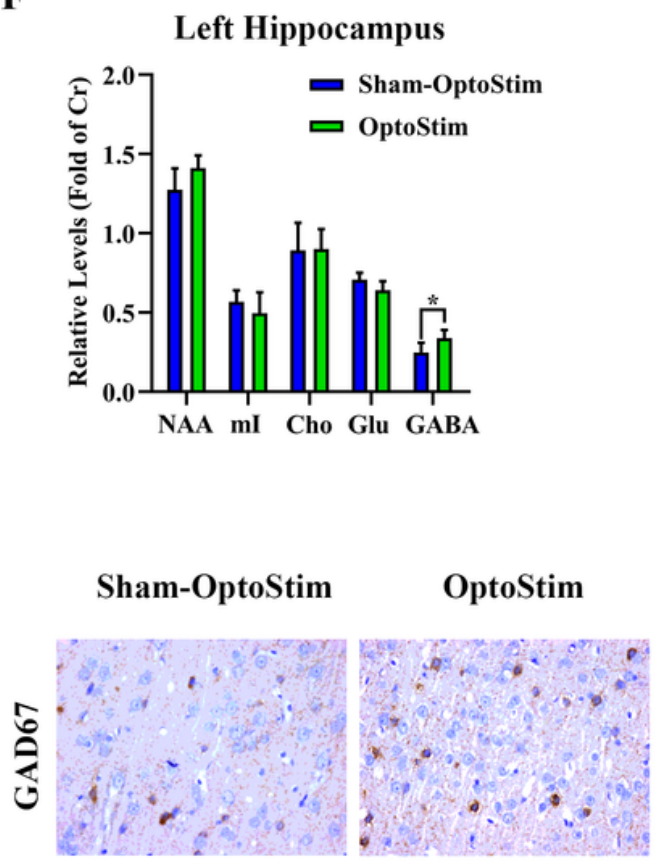

B

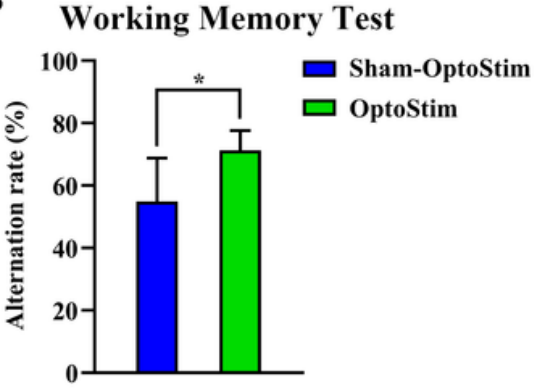

D

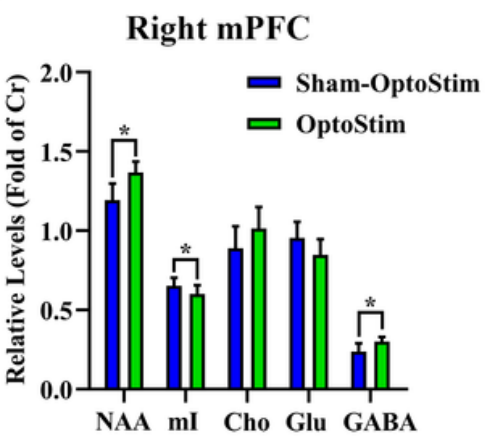

G

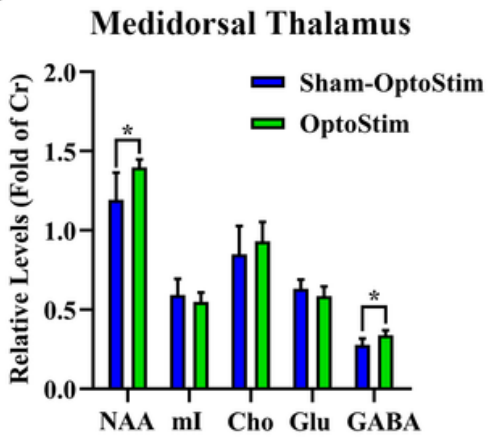

I

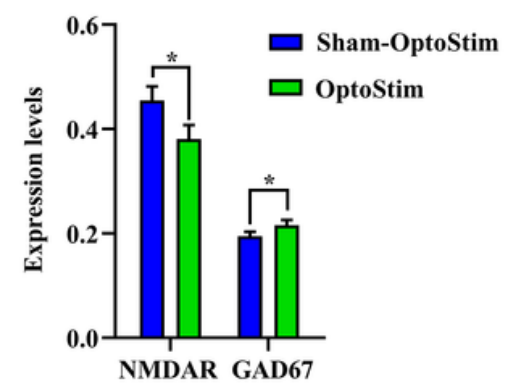

Figure 6

Optogenetics modulation of PV neurons in the MPFC was performed for neurochemical metabolic disorder and working memory impairment in rats with chronic cerebral ischemia. (A) The localization of virus injection and the expression of ChR2-eYFP (green) in the right mPFC. (B) The working memory was improved in the OptoStim group compared with the Sham-OptoStim group $(n=10)$. (C, D) Representative 1H-MRS graphs and statistical results in the right mPFC between the Sham-OptoStim group and OptoStim group $(n=10)$. $(E, F, G)$ The change of neurochemicals in the right hippocampus, left 
hippocampus and mediodorsal thalamus between the Sham-OptoStim group and OptoStim group, respectively $(n=5) .(H, I)$ The immunocytochemistry staining and statistical results of NMDAR and GAD67 $(\mathrm{n}=4)$. Abbreviation: PV, Parvalbumin; mPFC, medial prefrontal cortex; ChR2, channelrhodopsin; ml, myoinositol; Cho, choline; $\mathrm{Cr}$, creatine; Glu, glutamate; NAA, N-acetylaspartate, GABA, y-aminobutyric acid. OptoStim; Optogenetic Stimulation; NMDAR, N-methyl-D-aspartate receptor; GAD67, glutamic acid decarboxylase 67. ${ }^{*}, \mathrm{P}<0.05$.

\section{Supplementary Files}

This is a list of supplementary files associated with this preprint. Click to download.

- Fig.S1.tif

- Fig.S2.tif

- Fig.S3.tif

- Fig.S4.tif

- Fig.S5.tif

- Fig.S6.tif

- Fig.S7.tif

- Fig.S8.tif

- Fig.S9.tif

- Fig.S10.tif

- Fig.S11.tif

- Fig.S12.tif

- Fig.S13.tif

- Fig.S14.tif

- Fig.S15.tif

- Fig.S16.tif

- Fig.S17.tif

- Fig.S18.tif

- TableS1.docx 